\title{
WELL-POSEDNESS OF THE PLASMA-VACUUM INTERFACE PROBLEM FOR IDEAL INCOMPRESSIBLE MHD
}

\author{
YONGZHONG SUN, WEI WANG, AND ZHIFEI ZHANG
}

\begin{abstract}
Aвstract. In this paper, we prove the local well-posedness of plasma-vacuum interface problem for ideal incompressible magnetohydrodynamics under the stability condition: the magnetic field $\mathbf{h}$ and the vacuum magnetic field $\hat{\mathbf{h}}$ are non-collinear on the interface(i.e., $|\mathbf{h} \times \hat{\mathbf{h}}|>0$ ), which was introduced by Trakhinin as a stability condition for the compressible plasma-vacuum interface problem.
\end{abstract}

\section{INTRODUCTION}

1.1. Presentation of the problem. Magnetohydrodynamic (MHD) models describe macroscopic plasma phenomena, from laboratory research on thermonuclear fusion to plasma-astrophysics of the solar system. In the laboratory research, the main topic is magnetic plasma confinement for energy production by controlled thermonuclear reactions. The plasma-vacuum interface appears as a typical phenomenon when the plasma is separated by a vacuum from outside wall. The total pressure is balanced on the interface, while the normal part of the magnetic field vanishes and the tangent part may jump, thus forms a tangential discontinuity. Mathematically the plasmavacuum interface is formulated as a free boundary problem for the MHD system, see for example [8]. By ignoring the viscosity, the resistivity and heat conduction, we assume that the plasma fluid is ideal and incompressible. The evolution of the velocity $\mathbf{u}=\left(u^{1}, u^{2}, u^{3}\right)$, the magnetic field $\mathbf{h}=\left(h_{1}, h_{2}, h_{3}\right)$ and the total pressure $p$ is formulated by the following system of partial differential equations:

$$
\left\{\begin{array}{l}
\partial_{t} \mathbf{u}+\mathbf{u} \cdot \nabla \mathbf{u}-\mathbf{h} \cdot \nabla \mathbf{h}+\nabla p=0, \\
\operatorname{div} \mathbf{u}=0, \quad \operatorname{div} \mathbf{h}=0, \\
\partial_{t} \mathbf{h}+\mathbf{u} \cdot \nabla \mathbf{h}-\mathbf{h} \cdot \nabla \mathbf{u}=0 .
\end{array}\right.
$$

Here the total pressure $p=q+\frac{1}{2}|\mathbf{h}|^{2}$ with $q$ the fluid pressure. For technical reason, we consider the plasma-vacuum interface problem under a simplified configuration. Denote $\Omega=\mathbb{T}^{2} \times(-1,1)$ with the top/bottom boundary $\Gamma^{ \pm}=\mathbb{T}^{2} \times\{ \pm 1\}$ and assume that the plasma is initially confined in the domain

$$
\Omega_{f_{0}}^{-}=\left\{x=\left(x^{\prime}, x_{3}\right) \in \Omega \mid x_{3}<f_{0}\left(x^{\prime}\right)\right\}, x^{\prime}=\left(x_{1}, x_{2}\right) \in \mathbb{T}^{2},
$$

where $f_{0}\left(x^{\prime}\right)$ is a function defined on $\mathbb{T}^{2}$ and

$$
\Gamma_{f_{0}}:=\left\{x \in \Omega \mid x_{3}=f_{0}\left(x^{\prime}\right), x^{\prime} \in \mathbb{T}^{2}\right\}
$$

is the initial interface. Consequently,

$$
\Omega_{f_{0}}^{+}=\left\{x=\left(x^{\prime}, x_{3}\right) \in \Omega \mid x_{3}>f_{0}\left(x^{\prime}\right)\right\}
$$


is the region of the initial vacuum. After the initial time, the plasma evolves and the interface moves simultaneously. At the time $t>0$, let us assume the interface is represented as

$$
\Gamma_{f}=\Gamma_{f(t)}:=\left\{x \in \Omega \mid x_{3}=f\left(t, x^{\prime}\right), x^{\prime} \in \mathbb{T}^{2}\right\},
$$

and denote

$$
\begin{array}{ll}
\Omega_{f}^{-}=\Omega_{f(t)}^{-}=\left\{x=\left(x^{\prime}, x_{3}\right) \in \Omega \mid x_{3}<f\left(t, x^{\prime}\right)\right\}, & Q_{T}^{-}:=\cap_{t \in(0, T)}\{t\} \times \Omega_{f}^{-}, \\
\Omega_{f}^{+}=\Omega_{f(t)}^{+}=\left\{x=\left(x^{\prime}, x_{3}\right) \in \Omega \mid x_{3}>f\left(t, x^{\prime}\right)\right\}, & Q_{T}^{+}:=\cap_{t \in(0, T)}\{t\} \times \Omega_{f}^{+} .
\end{array}
$$

With these notations, the evolution of the plasma part is formulated as

$$
\begin{aligned}
& \partial_{t} \mathbf{u}+\mathbf{u} \cdot \nabla \mathbf{u}-\mathbf{h} \cdot \nabla \mathbf{h}+\nabla p=0 \text { in } Q_{T}^{-}, \\
& \operatorname{div} \mathbf{u}=0, \operatorname{div} \mathbf{h}=0 \text { in } Q_{T}^{-}, \\
& \partial_{t} \mathbf{h}+\mathbf{u} \cdot \nabla \mathbf{h}-\mathbf{h} \cdot \nabla \mathbf{u}=0 \text { in } Q_{T}^{-} .
\end{aligned}
$$

In the vacuum domain $\Omega_{f}^{+}$, we consider so-called pre-Maxwell dynamics. In such case, the magnetic field $\hat{\mathbf{h}}=\left(\hat{h}_{1}, \hat{h}_{2}, \hat{h}_{3}\right)$ is determined by the div-curl system:

$$
\operatorname{div} \hat{\mathbf{h}}=0, \quad \operatorname{curl} \hat{\mathbf{h}}=0 \quad \text { in } \Omega_{f}^{+} .
$$

The physical quantities of the plasma and the vacuum region are related by the pressure balance condition on the interface $\Gamma_{f}$ :

$$
p(t, x)=\frac{1}{2}|\hat{\mathbf{h}}(t, x)|^{2}, \quad(t, x) \in \Gamma_{f}
$$

as well as

$$
\mathbf{h} \cdot \mathbf{N}_{f}=0, \quad \hat{\mathbf{h}} \cdot \mathbf{N}_{f}=0, \quad(t, x) \in \Gamma_{f}
$$

Here

$$
\mathbf{N}_{f}=\left(N_{1}, N_{2}, N_{3}\right)=\left(-\partial_{1} f,-\partial_{2} f, 1\right)
$$

is the normal vector of $\Gamma_{f}$. As the interface moves with the fluid particles, its normal velocity $\partial_{t} f$ satisfies

$$
\partial_{t} f=\mathbf{u} \cdot \mathbf{N}_{f}
$$

Moreover, on the artificial boundaries $\Gamma^{ \pm}$, we prescribe the following boundary conditions:

$$
\begin{aligned}
& u_{3}=0, h_{3}=0 \text { on } \Gamma^{-}, \\
& \hat{\mathbf{h}} \times \mathbf{e}_{3}=\hat{\mathbf{J}} \text { on } \Gamma^{+},
\end{aligned}
$$

where $\mathbf{e}_{3}=(0,0,1)$ is the unit normal vector on $\Gamma^{+}$. Here to avoid trivial solution $\hat{\mathbf{h}}$ in the vacuum, a surface current $\hat{\mathbf{J}}=\left(\hat{J}_{1}, \hat{J}_{2}, \hat{J}_{3}\right)$ is added as an outer force term to the elliptic system (1.4). In real laboratory plasma, this surface current can be produced by a system of coils, see [ 8,15$]$. Finally, the system is supplemented with the initial data

$$
\mathbf{u}(0, x)=\mathbf{u}_{0}(x), \quad \mathbf{h}(0, x)=\mathbf{h}_{0}(x) \text { in } \Omega_{f_{0}}^{-}, \quad f\left(0, x_{1}, x_{2}\right)=f_{0}\left(x_{1}, x_{2}\right),
$$


which satisfy the following compatibility conditions:

$$
\left\{\begin{array}{l}
\operatorname{div} \mathbf{u}_{0}=0, \operatorname{div} \mathbf{h}_{0}=0 \text { in } \Omega_{f_{0}}^{-}, \\
\mathbf{h}_{0} \cdot \mathbf{N}_{f_{0}}=0 \text { on } \Gamma_{f_{0}}, u_{30}, h_{30}=0 \text { on } \Gamma^{-} .
\end{array}\right.
$$

From (1.9) and the fact that curl $\hat{\mathbf{h}}=0$, we also need compatibility conditions on the imposed surface current:

$$
\partial_{1} \hat{J}_{1}+\partial_{2} \hat{J}_{2}=0, \hat{J}_{3}=0 \text { on } \Gamma^{+} .
$$

Note that the initial magnetic field $\hat{\mathbf{h}}_{0}$ in the vacuum region is uniquely determined from $\Gamma_{f_{0}}, \mathbf{u}_{0}, \mathbf{h}_{0}$ and $\hat{\mathbf{J}}_{0}=\hat{\mathbf{J}}\left(0, x^{\prime}\right)$ by solving the following div-curl system:

$$
\left\{\begin{array}{l}
\operatorname{curl} \hat{\mathbf{h}}_{0}=0, \quad \operatorname{div} \hat{\mathbf{h}}_{0}=0 \text { in } \Omega_{f_{0}}^{+}, \\
\hat{\mathbf{h}}_{0} \cdot \mathbf{N}_{f_{0}}=0 \text { on } \Gamma_{f_{0}}, \quad \hat{\mathbf{h}}_{0} \times \mathbf{e}_{3}=\hat{\mathbf{J}}_{0} \text { on } \Gamma^{+} .
\end{array}\right.
$$

The solvability of this div-curl system will be shown in Section 4. Also note that since

$$
\partial_{t} \operatorname{div} \mathbf{h}+\mathbf{u} \cdot \nabla \operatorname{div} \mathbf{h}=0,
$$

the divergence free restriction on $\mathbf{h}$ is automatically satisfied if it holds for $\mathbf{h}_{0}$. Similar argument also yields $\mathbf{h} \cdot \mathbf{N}_{f}=0$ provided $\mathbf{h}_{0} \cdot \mathbf{N}_{f_{0}}=0$.

1.2. Backgrounds. In the absence of the magnetic field, the system is reduced to the incompressible Euler equations with a free boundary, which is so-called waterwave problem. In this case, it is well-known that a sufficient condition ensuring the well-posedness of the water-wave problem is the Taylor's sign condition:

$$
\frac{\partial p}{\partial \mathbf{N}} \leq-\epsilon<0 \quad \text { on } \Gamma_{f} .
$$

See [1, 6, 12, 13, 16, 23, 24, 25] and references therein. In fact, it is also necessary in the absence of surface tension [7].

The fact that the magnetic field has the stabilizing effect for the current-vortex sheet problem was found by physicists long before [2, 18]. In past decade, there are many works devoted to the well-posedness of the current-vortex sheet problem under a suitable stability condition [3, 5, 17, 19, 20, 22].

In [21], Trakhinin introduced the following stability condition for the linearized compressible plasma-vacuum interface problem:

$$
|\mathbf{h} \times \hat{\mathbf{h}}|>0 \text { on } \Gamma_{f} \text {. }
$$

Under this condition, Secchi and Trakhinin [15] proved the well-posedness of the compressible plasma-vacuum interface problem, and Morando, Trakhinin and Trebeschi [14] proved the well-posedness of the linearized incompressible plasma-vacuum interface problem. However, the well-posedness of nonlinear incompressible problem (i.e., the system (1.1)-(1.10) ) is still open under (1.15).

Motivated by works on the water-wave problem, Luo and Hao [10, 11] established a priori estimates for the incompressible plasma-vacuum interface problem under the Taylor's sign condition (1.14). Recently, Gu and Wang [9] proved the well-posedness of the incompressible plasma-vacuum problem under (1.14). Let us also mention that the well-posedness of the plasma-vacuum interface problem under (1.14) is still 
unknown when the vacuum magnetic field $\hat{\mathbf{h}}$ is non trivial. In [10, 11, 9], the authors only considered the case of $\hat{\mathbf{h}}=0$. This problem is also unsolved in the compressible case [21] .

1.3. Main result. The goal of this paper is to prove the well-posedness of the system (1.1)-(1.10) under the stability condition (1.15). This condition implies that $\mathbf{h}$ and $\hat{\mathbf{h}}$ are non-collinear everywhere on $\Gamma_{f}$, which means

$$
\inf _{x \in \Gamma_{f}} \inf _{\mathbf{q} \in T_{\Gamma_{f}}(x),|\mathbf{q}|=1}\left[(\mathbf{h}(x) \cdot \mathbf{q})^{2}+(\hat{\mathbf{h}}(x) \cdot \mathbf{q})^{2}\right]>0 .
$$

It will be shown in Section 5 that (1.16) implies that there exists a positive constant $c_{1}$ such that

$$
\Lambda(\mathbf{h}, \hat{\mathbf{h}}) \stackrel{\text { def }}{=} \inf _{x \in \Gamma_{f}} \inf _{\varphi_{1}^{2}+\varphi_{2}^{2}=1}\left[\left(h_{1} \varphi_{1}+h_{2} \varphi_{2}\right)^{2}+\left(\hat{h}_{1} \varphi_{1}+\hat{h}_{2} \varphi_{2}\right)^{2}\right] \geq c_{1}
$$

Our main result is stated as follows.

Theorem 1.1. Let $s \geq 3$ be an integer. Assume that

$$
\begin{aligned}
& f_{0} \in H^{s+\frac{1}{2}}\left(\mathbb{T}^{2}\right), \mathbf{u}_{0}, \mathbf{h}_{0} \in H^{s}\left(\Omega_{f_{0}}^{-}\right), \\
& \hat{\mathbf{J}} \in C\left(\left[0, T_{0}\right] ; H^{s-\frac{1}{2}}\left(\mathbb{T}^{2}\right)\right), \quad \partial_{t} \hat{\mathbf{J}} \in C\left(\left[0, T_{0}\right] ; H^{s-\frac{3}{2}}\left(\mathbb{T}^{2}\right)\right),
\end{aligned}
$$

which satisfies the compatibility conditions (1.11)-(1.12) and the stability condition:

$$
-\left(1-2 c_{0}\right) \leq f_{0} \leq\left(1-2 c_{0}\right), \quad \Lambda\left(\mathbf{h}_{0}, \hat{\mathbf{h}}_{0}\right) \geq 2 c_{1}
$$

for some $c_{0} \in\left(0, \frac{1}{2}\right)$ and $c_{1}>0$. Then there exists $T \in\left(0, T_{0}\right)$ such that the system (1.1)-(1.10) admits a unique solution $(f, \mathbf{u}, \mathbf{h}, \hat{\mathbf{h}})$ in $[0, T]$ such that

$$
\begin{aligned}
& f \in L^{\infty}\left(0, T ; H^{s+\frac{1}{2}}\left(\mathbb{T}^{2}\right)\right), \mathbf{u}, \mathbf{h} \in L^{\infty}\left(0, T ; H^{s}\left(\Omega_{f}^{-}\right)\right), \hat{\mathbf{h}} \in L^{\infty}\left(0, T ; H^{s}\left(\Omega_{f}^{+}\right)\right) \\
& -\left(1-c_{0}\right) \leq f \leq\left(1-c_{0}\right), \Lambda(\mathbf{h}, \hat{\mathbf{h}}) \geq c_{1}
\end{aligned}
$$

The idea of the proof is motivated by our recent work on nonlinear stability of incompressible current-vortex sheet problem [17]. The key ingredient is to derive an evolution equation of the scaled normal velocity $\mathbf{u} \cdot \mathbf{N}$ rather than the usual normal component of velocity on the interface. By some tricky observations, we find in present case that the evolution equation of the height function of the interface takes as follows

$$
D_{t}^{2} f=\sum_{i, j=1,2}\left(\underline{h_{i}} \underline{h_{j}}+\underline{\hat{h}_{i}} \underline{\hat{h}_{j}}\right) \partial_{i} \partial_{j} f+\text { L.O.T. }
$$

where $D_{t} f=\partial_{t} f+u_{1} \partial_{1} f+u_{2} \partial_{2} f, g$ denotes the trace of $g$ on the interface, and L.O.T. denotes the lower order terms. Now, the stability condition (1.17) ensures that the equation (1.21) is strictly hyperbolic. Indeed, the principal symbol of the operator $\left.-\sum_{i, j=1,2} \underline{\left(h_{i}\right.} \underline{h_{j}}+\underline{\hat{h}_{i}} \underline{\hat{h}_{j}}\right) \partial_{i} \partial_{j}$ is

$$
\left(\underline{h_{i}} \xi_{i}\right)^{2}+\left(\underline{\hat{h}_{i}} \xi_{i}\right)^{2} \geq c_{1}|\xi|^{2} .
$$


The motion of the fluid and the magnetic field will be described by the following vorticity and current system:

$$
\left\{\begin{array}{l}
\partial_{t} \boldsymbol{\omega}+\mathbf{u} \cdot \nabla \boldsymbol{\omega}-\mathbf{h} \cdot \nabla \boldsymbol{\xi}=\boldsymbol{\omega} \cdot \nabla \mathbf{u}-\boldsymbol{\xi} \cdot \nabla \mathbf{h} \text { in } Q_{T}^{-}, \\
\partial_{t} \boldsymbol{\xi}+\mathbf{u} \cdot \nabla \boldsymbol{\xi}-\mathbf{h} \cdot \nabla \boldsymbol{\omega}=\boldsymbol{\xi} \cdot \nabla \mathbf{u}-\boldsymbol{\omega} \cdot \nabla \mathbf{h}-2 \nabla u_{i} \times \nabla h_{i} \text { in } Q_{T}^{-}
\end{array}\right.
$$

where $\omega=\nabla \times \mathbf{u}, \boldsymbol{\xi}=\nabla \times \mathbf{h}$. With the vorticity and current, the velocity and magnetic field can be recovered by solving two div-curl systems defined on the fluid domain and the vacuum domain respectively. For the fluid domain, we need to solve a divcurl system with given normal component on the boundaries, which is the same as in [17]. For the vacuum domain, we need to solve another div-curl system for $\hat{\mathbf{h}}$ :

$$
\left\{\begin{array}{l}
\operatorname{div} \hat{\mathbf{h}}=0, \quad \operatorname{curl} \hat{\mathbf{h}}=0 \text { in } \Omega_{f}^{+}, \\
\hat{\mathbf{h}} \cdot \mathbf{n}_{f}=\hat{\vartheta} \text { on } \Gamma_{f}, \quad \hat{\mathbf{h}} \times \mathbf{e}_{3}=\hat{\mathbf{J}} \text { on } \Gamma^{+} .
\end{array}\right.
$$

The main difference of these two system is the boundary condition on the fixed boundary. We state the solvability and estimates of solutions to the div-curl systems in Section 4.

The construction of the approximate solution is completed by introducing the suitable linearization of the system and the iteration map. It can be proved that the constructed approximate sequence is a Cauchy sequence, and the limit system is equivalent to the origin system.

The rest of this paper is organized as follows. In Section 2, we introduce the harmonic coordinate and preliminary results on the harmonic extensions and Dirichlet to Neumann operators. In Section 3, the system is replaced by a new formulation. In Section 4, two div-curl systems are solved. In Section 5, we establish the uniform estimates for the linearized system. Section 6 are devoted to proving existence (and uniqueness) of the solution.

\section{Harmonic Coordinate and Dirichlet-Neumann Operator}

In this section, we recall some facts and well-known results on the reference domain, the harmonic coordinate and Dirichlet-Neumann operators.

We first introduce some notations used throughout this paper. The coordinate in the fluid region is denoted as $x=\left(x_{1}, x_{2}, x_{3}\right)$ or $y=\left(y_{1}, y_{2}, y_{3}\right)$, while $x^{\prime}=\left(x_{1}, x_{2}\right)$ or $y^{\prime}=\left(y_{1}, y_{2}\right)$ is the natural coordinates on the interface or on the top/bottom boundary $\Gamma^{ \pm}$. For a function $g: \Omega \rightarrow \mathbb{R}$, we denote $\nabla g=\left(\partial_{1} g, \partial_{2} g, \partial_{3} g\right)$ and for a function $\eta: \mathbb{T}^{2} \rightarrow \mathbb{R}, \nabla \eta=\left(\partial_{1} \eta, \partial_{2} \eta\right)$. The trace on $\Gamma_{f}$ for a function $g: \Omega_{f}^{ \pm} \rightarrow \mathbb{R}$ is denoted by $g$. Thus, for $i=1,2$,

$$
\left(\partial_{i} g\right)\left(x^{\prime}\right)=\partial_{i} g\left(x^{\prime}, f\left(x^{\prime}\right)\right)+\partial_{3} g\left(x^{\prime}, f\left(x^{\prime}\right)\right) \partial_{i} f\left(x^{\prime}\right)=\underline{\partial_{i} g}+\underline{\partial_{3} g} \partial_{i} f\left(x^{\prime}\right) .
$$

Finally, the Sobolev norm in $\Omega_{f}^{ \pm}$is denoted as $\|\cdot\|_{H^{k}\left(\Omega_{f}^{ \pm}\right)}$and $\|\cdot\|_{H^{k}}$ is the Sobolev norm in $\mathbb{T}^{2}$.

To solve the free boundary problem, we introduce a fixed reference domain. Let $\Gamma_{*}$ be a fixed graph given by

$$
\Gamma_{*}=\left\{\left(y_{1}, y_{2}, y_{3}\right): y_{3}=f_{*}\left(y_{1}, y_{2}\right)\right\} .
$$


The reference domain $\Omega_{*}^{ \pm}$is given by

$$
\Omega_{*}=\mathbb{T}^{2} \times(-1,1), \quad \Omega_{*}^{ \pm}=\left\{y \in \Omega_{*} \mid y_{3} \gtrless f_{*}\left(y^{\prime}\right)\right\} .
$$

We will seek a free boundary lying in a neighborhood of the reference domain. To this end, we define

$$
\Upsilon(\delta, k) \stackrel{\text { def }}{=}\left\{f \in H^{k}\left(\mathbb{T}^{2}\right):\left\|f-f_{*}\right\|_{H^{k}\left(\mathbb{T}^{2}\right)} \leq \delta\right\} .
$$

For $f \in \Upsilon(\delta, k)$, the graph $\Gamma_{f}$ is defined as

$$
\Gamma_{f} \stackrel{\text { def }}{=}\left\{x \in \Omega \mid x_{3}=f\left(t, x^{\prime}\right), x^{\prime} \in \mathbb{T}^{2}\right\},
$$

and use the notations $\Omega_{f}^{ \pm}, \mathbf{N}_{f}, \Gamma^{ \pm}$, etc., as in Section 1 ,

Remark 2.1. Since we intend to solve the plasma-vacuum interface problem locally in time, a natural choice of $\Gamma_{*}$ would be certainly the initial interface $\Gamma_{0}$.

To handle the plasma-vacuum interface problem, we need to introduce different Dirichlet-Neumman operators on $\Omega_{f}^{ \pm}$. For a function $\psi\left(x^{\prime}\right)=\psi\left(x_{1}, x_{2}\right) \in H^{s}\left(\mathbb{T}^{2}\right)$, its harmonic extension from $\Gamma_{f}$ to $\Omega_{f}^{ \pm}$is denoted as $\mathcal{H}_{f}^{ \pm} \psi$, i.e.,

$$
\left\{\begin{array}{l}
\Delta \mathcal{H}_{f}^{ \pm} \psi=0 \text { in } \Omega_{f}^{ \pm}, \\
\left(\mathcal{H}_{f}^{ \pm} \psi\right)\left(x^{\prime}, f\left(x^{\prime}\right)\right)=\psi\left(x^{\prime}\right), x^{\prime} \in \mathbb{T}^{2}, \\
\partial_{3}\left(\mathcal{H}_{f}^{ \pm} \psi\right)\left(x^{\prime}, \pm 1\right)=0, x^{\prime} \in \mathbb{T}^{2}
\end{array}\right.
$$

Moreover, for a function $g$ defined in $\Omega_{f}^{ \pm}$, we denote $\widehat{\mathcal{H}}_{f}^{ \pm} g$ as a harmonic function in $\Omega_{f}^{ \pm}$such that

$$
\left\{\begin{array}{l}
\Delta \widehat{\mathcal{H}}_{f}^{ \pm} g=0 \text { in } \Omega_{f}^{ \pm}, \\
\left(\widehat{\mathcal{H}}_{f}^{ \pm} g\right)\left(x^{\prime}, f\left(x^{\prime}\right)\right)=g\left(x^{\prime}, f\left(x^{\prime}\right)\right), x^{\prime} \in \mathbb{T}^{2}, \\
\partial_{3}\left(\widehat{\mathcal{H}}_{f}^{ \pm} g\right)\left(x^{\prime}, \pm 1\right)=\partial_{3} g\left(x^{\prime}, \pm 1\right), x^{\prime} \in \mathbb{T}^{2} .
\end{array}\right.
$$

The Dirichlet-Neumann (D-N in the following context) operators are defined as

$$
\mathcal{N}_{f}^{ \pm} \psi=\left.\mp \mathbf{N}_{f} \cdot \nabla \mathcal{H}_{f}^{ \pm} \psi\right|_{\Gamma_{f}}, \quad \widehat{\mathcal{N}}_{f}^{ \pm} g=\left.\mp \mathbf{N}_{f} \cdot \nabla \widehat{\mathcal{H}}_{f}^{ \pm} g\right|_{\Gamma_{f}} .
$$

For a function $g$ defined in $\Omega_{f}^{+}$, we denote

$$
\overline{\mathcal{N}}_{f} g=\widehat{\mathcal{N}}_{f}^{+} g-\mathcal{N}_{f}^{-} \underline{g} .
$$

Given $f \in \Upsilon(\delta, k)$, a map (harmonic coordinate) $\Phi_{f}^{ \pm}$from $\Omega_{*}^{ \pm}$to $\Omega_{f}^{ \pm}$is defined by harmonic extension:

$$
\left\{\begin{array}{l}
\Delta_{y} \Phi_{f}^{ \pm}=0 \text { in } \Omega_{*}^{ \pm}, \\
\Phi_{f}^{ \pm}\left(y^{\prime}, f_{*}\left(y^{\prime}\right)\right)=\left(y^{\prime}, f\left(y^{\prime}\right)\right), y^{\prime} \in \mathbb{T}^{2}, \\
\Phi_{f}^{ \pm}\left(y^{\prime}, \pm 1\right)=\left(y^{\prime}, \pm 1\right), y^{\prime} \in \mathbb{T}^{2} .
\end{array}\right.
$$


Given $\Gamma_{*}$, there exists $\delta_{0}=\delta_{0}\left(\left\|f_{*}\right\|_{W^{1, \infty}}\right)>0$ so that $\Phi_{f}^{ \pm}$is a bijection whenever $\delta \leq \delta_{0}$. Thus, there exists an inverse map $\Phi_{f}^{ \pm-1}$ from $\Omega_{f}^{ \pm}$to $\Omega_{*}^{ \pm}$such that

$$
\Phi_{f}^{ \pm-1} \circ \Phi_{f}^{ \pm}=\operatorname{Id}_{\Omega_{*}^{ \pm}}, \Phi_{f}^{ \pm} \circ \Phi_{f}^{ \pm-1}=\operatorname{Id}_{\Omega_{f}^{ \pm}} .
$$

We have the following properties of harmonic coordinates, see [17] for example.

Lemma 2.1. Let $f \in \Upsilon\left(\delta_{0}, s-\frac{1}{2}\right)$ for $s \geq 3$. There exists a constant $C$ depending only on $\delta_{0}$ and $\left\|f_{*}\right\|_{H^{s-\frac{1}{2}}}$ so that

1. If $u \in H^{\sigma}\left(\Omega_{f}^{ \pm}\right)$for $\sigma \in[0, s]$, then

$$
\left\|u \circ \Phi_{f}^{ \pm}\right\|_{H^{\sigma}\left(\Omega_{*}^{ \pm}\right)} \leq C\|u\|_{H^{\sigma}\left(\Omega_{f}^{ \pm}\right)} .
$$

2. If $u \in H^{\sigma}\left(\Omega_{*}^{ \pm}\right)$for $\sigma \in[0, s]$, then

$$
\left\|u \circ \Phi_{f}^{ \pm-1}\right\|_{H^{\sigma}\left(\Omega_{f}^{ \pm}\right)} \leq C\|u\|_{H^{\sigma}\left(\Omega_{*}^{ \pm}\right)} .
$$

3. If $u, v \in H^{\sigma}\left(\Omega_{*}^{ \pm}\right)$for $\sigma \in[2, s]$, then

$$
\left\|(u v) \circ \Phi_{f}^{ \pm-1}\right\|_{H^{\sigma}\left(\Omega_{f}^{ \pm}\right)} \leq C\|u\|_{H^{\sigma}\left(\Omega_{*}^{ \pm}\right)}\|v\|_{H^{\sigma}\left(\Omega_{*}^{ \pm}\right)} .
$$

Proposition 2.1. Let $s \geq 3$ be an integer. If $f \in H^{s+\frac{1}{2}}\left(\mathbb{T}^{2}\right)$, then we have

$$
\left\|\overline{\mathcal{N}}_{f} g\right\|_{H^{s-\frac{1}{2}}} \leq C\left(\|f\|_{H^{s+\frac{1}{2}}}\right)\|g\|_{H^{s}\left(\Omega_{f}^{+}\right)} .
$$

Proof. We write

$$
\overline{\mathcal{N}}_{f} g=\left(\widehat{\mathcal{N}}_{f}^{+} g-\mathcal{N}_{f}^{+} g\right)+\left(\mathcal{N}_{f}^{+} g-\mathcal{N}_{f}^{-} g\right) .
$$

The corresponding estimate for the second term on the right hand side has been shown in the appendix of [17]. The first term in fact satisfies

$$
\left\|\widehat{\mathcal{N}}_{f}^{+} g-\mathcal{N}_{f}^{+} g\right\|_{H^{s-\frac{1}{2}}} \leq C\left(\|f\|_{H^{s+\frac{1}{2}}}\right)\|g\|_{H^{2}\left(\Omega_{f}^{+}\right)} .
$$

To prove this, we first note that

$$
\overline{\mathcal{H}}_{f}^{+} g=\widehat{\mathcal{H}}_{f}^{+} g-\mathcal{H}_{f}^{+} g
$$

satisfies

$$
\Delta \overline{\mathcal{H}}_{f}^{+} g=0 \text { in } \Omega_{f}^{+}, \overline{\mathcal{H}}_{f}^{+} g=0 \text { on } \Gamma_{f}, \partial_{3} \overline{\mathcal{H}}_{f}^{+} g=\partial_{3} g \text { on } \Gamma^{+} .
$$

It follows that

$$
\begin{gathered}
\int_{\Omega_{f}^{+}}\left|\nabla \overline{\mathcal{H}}_{f}^{+} g\right|^{2} d x=\int_{\Gamma^{+}}\left(\overline{\mathcal{H}}_{f}^{+} g\right)\left(\partial_{3} g\right) d x^{\prime} \leq\left\|\overline{\mathcal{H}}_{f}^{+} g\right\|_{L^{2}}\left\|\partial_{3} g\right\|_{L^{2}} \\
\leq C \mid\left\|\overline{\mathcal{H}}_{f}^{+} g\right\|_{H^{1}\left(\Omega_{f}^{+}\right)}\|g\|_{H^{2}\left(\Omega_{f}^{+}\right)} \leq C\left\|\nabla \overline{\mathcal{H}}_{f}^{+} g\right\|_{L^{2}\left(\Omega_{f}^{+}\right)}\|g\|_{H^{2}\left(\Omega_{f}^{+}\right)},
\end{gathered}
$$

where in the last inequality we applied Poincaré's inequality to $\overline{\mathcal{H}}_{f}^{+} g$. On the other hand, according to standard interior (and boundary near $\Gamma_{f}$ ) elliptic estimates, we have

$$
\left\|\overline{\mathcal{H}}_{f}^{+} g\right\|_{H^{s}\left(\widetilde{\Omega_{f}^{+}}\right)} \leq C\left(\|f\|_{H^{s+\frac{1}{2}}}\right)\left\|\overline{\mathcal{H}}_{f}^{+} g\right\|_{H^{1}\left(\Omega_{f}^{+}\right)} .
$$

Here $\widetilde{\Omega_{f}^{+}}$is any sub-domain of $\Omega_{f}^{+}$away from $\Gamma^{+}$, such as

$$
\widetilde{\Omega_{f}^{+}}=\left\{x \in \Omega_{f}^{+} \mid f\left(x^{\prime}\right)<x_{3}<1-\epsilon_{0}\right\} \text { for sufficiently small } \epsilon_{0} .
$$

We thus conclude the proof of (2.8), and then (2.7) follows. 
Finally we introduce a commutator estimates that will be used frequently.

Lemma 2.2. For $s>2$, we have

$$
\left\|\left[a,\langle\nabla\rangle^{s}\right] u\right\|_{L^{2}} \leq C\|a\|_{H^{s}}\|u\|_{H^{s-1}} .
$$

Here $\langle\nabla\rangle^{s}$ is the s-order derivatives on $\mathbb{T}^{2}$ defined as follows

$$
\widehat{\langle\nabla\rangle^{s}} f(\mathbf{k})=\left(1+|\mathbf{k}|^{2}\right)^{\frac{s}{2}} \hat{f}(\mathbf{k}), \quad \mathbf{k}=\left(k_{1}, k_{2}\right), k_{1}, k_{2} \in \mathbb{Z} .
$$

\section{Reformulation OF THE PROBLEM}

In this section, we replace the system (1.1)-11.10) by an equivalent formulation, which consists of the (evolution) equations of the following quantities:

- the height function of the interface: $f$;

- the scaled normal velocity on the interface: $\theta=\mathbf{u} \cdot \mathbf{N}_{f}$;

- the vorticity and current in the fluid region: $\omega=\nabla \times \mathbf{u}, \boldsymbol{\xi}=\nabla \times \mathbf{h}$;

- the average of tangential part of velocity and magnetic field on the fixed bottom boundary:

$$
\beta_{i}(t)=\int_{\mathbb{T}^{2}} u_{i}\left(t, x^{\prime},-1\right) d x^{\prime}, \gamma_{i}(t)=\int_{\mathbb{T}^{2}} h_{i}\left(t, x^{\prime},-1\right) d x^{\prime}, i=1,2 .
$$

To simplify notations, from now on we drop the minus superscript "'-". Hence,

$$
\Omega_{f}=\Omega_{f}^{-}, \Gamma=\Gamma^{-}, \mathcal{N}_{f}=\mathcal{N}_{f}^{-}, \mathcal{H}_{f}=\mathcal{H}_{f}^{-}, \text {etc. } .
$$

\subsection{Evolution of the interface and the scaled normal velocity. Let}

$$
\theta\left(t, x^{\prime}\right)=\mathbf{u}\left(t, x^{\prime}, f\left(t, x^{\prime}\right)\right) \cdot \mathbf{N}_{f}\left(t, x^{\prime}\right) .
$$

Then we have

$$
\partial_{t} f\left(t, x^{\prime}\right)=\theta\left(t, x^{\prime}\right) .
$$

Clearly, $\left(1+|\nabla f|^{2}\right)^{-1 / 2} \theta$ is the normal component of the fluid velocity on the interface.

According to (2.1), for a vector field $\mathbf{v}=\left(v_{1}, v_{2}, v_{3}\right)$ defined in $\Omega_{f}^{+}$or $\Omega_{f}$ we calculate $\underline{\mathbf{v} \cdot \nabla \mathbf{v}} \cdot \mathbf{N}_{f}$ as follows:

$$
\begin{aligned}
& (\underline{\mathbf{v} \cdot \nabla \mathbf{v}}) \cdot \mathbf{N}_{f}=\underline{v_{1}} \underline{\partial_{1} v_{j}} N_{j}+\underline{v_{2}} \underline{\partial_{2} v_{j}} N_{j}+\underline{v_{3}} \underline{\partial_{3} v_{j}} N_{j} \\
& =\underline{v_{1}} \partial_{1} \underline{v_{j}} N_{j}+\underline{v_{2}} \partial_{2} \underline{v_{j}} N_{j}+\left(\underline{\mathbf{v}} \cdot \mathbf{N}_{f}\right)\left(\underline{\partial_{3} \mathbf{v}} \cdot \mathbf{N}_{f}\right) \\
& =\underline{v_{1}} \partial_{1}\left(\underline{\mathbf{v}} \cdot \mathbf{N}_{f}\right)+\underline{v_{2}} \partial_{2}\left(\underline{\mathbf{v}} \cdot \mathbf{N}_{f}\right)-\underline{v_{1}} \underline{v_{j}} \partial_{1} N_{j}-\underline{v_{2}} \underline{v_{j}} \partial_{2} N_{j}+\left(\underline{\mathbf{v}} \cdot \mathbf{N}_{f}\right)\left(\underline{\partial_{3} \mathbf{v}} \cdot \mathbf{N}_{f}\right) .
\end{aligned}
$$

Hereafter we use Einstein's notation of summation for repeated indices $i, j=1,2,3$ as well as summation on $i, j=1,2$ in case of making no confusion. From the calculations above, we obtain the following lemma.

Lemma 3.1. For a vector $\mathbf{v}=\left(v_{1}, v_{2}, v_{3}\right)$ defined in $\Omega_{f}^{+}$or $\Omega_{f}$,

$$
\begin{aligned}
& (\underline{\mathbf{v} \cdot \nabla \mathbf{v}}) \cdot \mathbf{N}_{f}-\left(\underline{\partial_{3} \mathbf{v}} \cdot \mathbf{N}_{f}\right)\left(\underline{\mathbf{v}} \cdot \mathbf{N}_{f}\right) \\
& =\underline{v_{1}} \partial_{1}\left(\underline{\mathbf{v}} \cdot \mathbf{N}_{f}\right)+\underline{v_{2}} \partial_{2}\left(\underline{\mathbf{v}} \cdot \mathbf{N}_{f}\right)+\sum_{i, j=1,2} \underline{v_{i}} \underline{v_{j}} \partial_{i} \partial_{j} f .
\end{aligned}
$$


By restricting the equation (1.1) to $\Gamma_{f}$ and taking inner product with $\mathbf{N}_{f}$, we deduce from Lemma3.1 (recall $\mathbf{h} \cdot \mathbf{N}_{f}=0$ on $\Gamma_{f}$ ) that

$$
\begin{aligned}
\partial_{t} \theta= & \left(\partial_{t} \mathbf{u}+\partial_{3} \mathbf{u} \partial_{t} f\right) \cdot \mathbf{N}_{f}+\left.\mathbf{u} \cdot \partial_{t} \mathbf{N}_{f}\right|_{x_{3}=f\left(t, x^{\prime}\right)} \\
= & \left(-\mathbf{u} \cdot \nabla \mathbf{u}+\mathbf{h} \cdot \nabla \mathbf{h}-\nabla p+\partial_{3} \mathbf{u} \partial_{t} f\right) \cdot \mathbf{N}_{f}-\left.\mathbf{u} \cdot\left(\partial_{1} \partial_{t} f, \partial_{2} \partial_{t} f, 0\right)\right|_{x_{3}=f\left(t, x^{\prime}\right)} \\
= & \left(-(\mathbf{u} \cdot \nabla) \mathbf{u}+\partial_{3} \mathbf{u}\left(\mathbf{u} \cdot \mathbf{N}_{f}\right)\right) \cdot \mathbf{N}_{f}+(\mathbf{h} \cdot \nabla) \mathbf{h} \cdot \mathbf{N}_{f} \\
& -\mathbf{N}_{f} \cdot \nabla p-\left.\mathbf{u} \cdot\left(\partial_{1} \theta, \partial_{2} \theta, 0\right)\right|_{x_{3}=f\left(t, x^{\prime}\right)} \\
= & -2\left(\underline{u_{1}} \partial_{1} \theta+\underline{u_{2}} \partial_{2} \theta\right)-\mathbf{N}_{f} \cdot \underline{\nabla p}-\sum_{i, j=1,2} \underline{u_{i}} \underline{u_{j}} \partial_{i} \partial_{j} f+\sum_{i, j=1,2} \underline{h_{i}} \underline{h_{j}} \partial_{i} \partial_{j} f .
\end{aligned}
$$

To give the trace of the pressure $p$ on $\Gamma_{f}$, we first take divergence to (1.1) to yield

$$
\Delta p=\operatorname{tr}(\nabla \mathbf{h} \nabla \mathbf{h})-\operatorname{tr}(\nabla \mathbf{u} \nabla \mathbf{u})
$$

Let $p_{\mathbf{v}_{1}, \mathbf{v}_{2}}$ be the solution of the following mixed boundary value problem:

$$
\left\{\begin{array}{l}
\Delta p_{\mathbf{v}_{1}, \mathbf{v}_{2}}=-\operatorname{tr}\left(\nabla \mathbf{v}_{1} \nabla \mathbf{v}_{2}\right) \text { in } \Omega_{f}, \\
p_{\mathbf{v}_{1}, \mathbf{v}_{2}}=0 \text { on } \Gamma_{f}, \mathbf{e}_{3} \cdot \nabla p_{\mathbf{v}_{1}, \mathbf{v}_{2}}=0 \text { on } \Gamma .
\end{array}\right.
$$

Since $\underline{p}=\left.p\right|_{\Gamma_{f}}=\left.\frac{1}{2}|\hat{\mathbf{h}}|^{2}\right|_{\Gamma_{f}}$, we obtain the following representation formula for the pressure $p$ :

$$
p=\mathcal{H}_{f} \underline{p}+p_{\mathbf{u}, \mathbf{u}}-p_{\mathbf{h}, \mathbf{h}}=\frac{1}{2} \mathcal{H}_{f}|\underline{\hat{\mathbf{h}}}|^{2}+p_{\mathbf{u}, \mathbf{u}}-p_{\mathbf{h}, \mathbf{h}} .
$$

It follows from (3.4) that

$$
\begin{aligned}
\partial_{t} \theta= & -2\left(\underline{u_{1}} \partial_{1} \theta+\underline{u_{2}} \partial_{2} \theta\right)-\sum_{i, j=1,2}\left(\underline{u_{i}} \underline{u_{j}}-\underline{h_{i}} \underline{h_{j}}\right) \partial_{i} \partial_{j} f \\
& -\frac{1}{2} \mathcal{N}_{f}|\underline{\hat{\mathbf{h}}}|^{2}-\mathbf{N}_{f} \cdot \underline{\nabla\left(p_{\mathbf{u}, \mathbf{u}}-p_{\mathbf{h}, \mathbf{h}}\right)} .
\end{aligned}
$$

Note that

$$
\begin{aligned}
-\mathcal{N}_{f}|\underline{\hat{\mathbf{h}}}|^{2} & =\left(\widehat{\mathcal{N}}_{f}^{+}-\mathcal{N}_{f}\right)|\hat{\mathbf{h}}|^{2}-\mathbf{N}_{f} \cdot \underline{\nabla\left(|\hat{\mathbf{h}}|^{2}-\widehat{\mathcal{H}}_{f}^{+}|\hat{\mathbf{h}}|^{2}\right)}+\mathbf{N}_{f} \cdot \underline{\nabla\left(|\hat{\mathbf{h}}|^{2}\right)} \\
& =\overline{\mathcal{N}}_{f}|\hat{\mathbf{h}}|^{2}-\mathbf{N}_{f} \cdot \underline{\nabla\left(|\hat{\mathbf{h}}|^{2}-\hat{\mathcal{H}}_{f}^{+}|\hat{\mathbf{h}}|^{2}\right)+\mathbf{N}_{f} \cdot \underline{\nabla\left(|\hat{\mathbf{h}}|^{2}\right)} .}
\end{aligned}
$$

Furthermore, by Lemma 3.1 ,

$$
\sum_{i, j=1,2} \underline{\hat{h}_{i}} \underline{\hat{h}_{j}} \partial_{i} \partial_{j} f=\underline{(\hat{\hat{\mathbf{h}}} \cdot \nabla \hat{\mathbf{h}})} \cdot \mathbf{N}_{f}=\hat{h}_{i} \partial_{i} \hat{h}_{j} N_{j}=\hat{h}_{i} \partial_{j} \hat{h}_{i} N_{j}=\frac{1}{2} \mathbf{N}_{f} \cdot \nabla|\hat{\mathbf{h}}|^{2},
$$

where we used curl $\hat{\mathbf{h}}=0$. From (3.8)-(3.10), we finally obtain

$$
\begin{aligned}
\partial_{t} \theta= & -2\left(\underline{u_{1}} \partial_{1} \theta+\underline{u_{2}} \partial_{2} \theta\right)-\sum_{i, j=1,2}\left(\underline{u_{i}} \underline{u_{j}}-\underline{h_{i}} \underline{h_{j}}-\underline{\hat{h}_{i}} \underline{\hat{h}_{j}}\right) \partial_{i} \partial_{j} f \\
& -\mathbf{N}_{f} \cdot \underline{\nabla\left(p_{\mathbf{u}, \mathbf{u}}-p_{\mathbf{h}, \mathbf{h}}\right)}-\frac{1}{2} \mathbf{N}_{f} \cdot \underline{\nabla\left(|\hat{\mathbf{h}}|^{2}-\hat{\mathcal{H}}_{f}^{+}|\hat{\mathbf{h}}|^{2}\right)}+\frac{1}{2} \overline{\mathcal{N}}_{f}|\hat{\mathbf{h}}|^{2} .
\end{aligned}
$$




\subsection{The equations for the vorticity and current. Let}

$$
\omega=\nabla \times \mathbf{u}, \quad \xi=\nabla \times \mathbf{h}
$$

be the vorticity and current in $\Omega_{f}$ respectively. Then $\omega, \xi$ satisfy

$$
\begin{aligned}
& \partial_{t} \boldsymbol{\omega}+\mathbf{u} \cdot \nabla \boldsymbol{\omega}-\mathbf{h} \cdot \nabla \boldsymbol{\xi}=\boldsymbol{\omega} \cdot \nabla \mathbf{u}-\boldsymbol{\xi} \cdot \nabla \mathbf{h} \text { in } Q_{T}, \\
& \partial_{t} \boldsymbol{\xi}+\mathbf{u} \cdot \nabla \boldsymbol{\xi}-\mathbf{h} \cdot \nabla \boldsymbol{\omega}=\boldsymbol{\xi} \cdot \nabla \mathbf{u}-\boldsymbol{\omega} \cdot \nabla \mathbf{h}-2 \nabla u_{i} \times \nabla h_{i} \text { in } Q_{T} .
\end{aligned}
$$

Here we used the fact that

$$
\begin{aligned}
& \varepsilon^{i j k} \partial_{j} u_{l} \partial_{l} h_{k}-\varepsilon^{i j k} \partial_{j} h_{l} \partial_{l} u_{k} \\
& =\varepsilon^{i j k} \partial_{j} u_{l}\left(\partial_{l} h_{k}-\partial_{k} h_{l}\right)+\varepsilon^{i j k} \partial_{j} u_{l} \partial_{k} h_{l}-\varepsilon^{i j k} \partial_{j} h_{l} \partial_{k} u_{l}+\varepsilon^{i j k} \partial_{j} h_{l}\left(\partial_{k} u_{l}-\partial_{l} u_{k}\right) \\
& =-\boldsymbol{\xi} \cdot \nabla \mathbf{u}+\omega \cdot \nabla \mathbf{h}-2 \nabla u_{i} \times \nabla h_{i} .
\end{aligned}
$$

As in [17], to uniquely recover a divergence free vector field from its curl and normal component(on the bottom boundary) in $\Omega_{f}$, we need to prescribe the mean value of its tangential components on the bottom boundary. To this end, let

$$
\beta_{i}(t)=\int_{\mathbb{T}^{2}} u_{i}\left(t, x^{\prime},-1\right) d x^{\prime}, \gamma_{i}(t)=\int_{\mathbb{T}^{2}} h_{i}\left(t, x^{\prime},-1\right) d x^{\prime}, i=1,2 .
$$

Thanks to $u_{3}\left(t, x^{\prime},-1\right) \equiv 0$, we deduce that for $i=1,2$,

$$
\partial_{t} u_{i}+u_{j} \partial_{j} u_{i}-h_{j} \partial_{j} h_{i}-\partial_{i} p=0 \text { on } \Gamma
$$

which yields

$$
\partial_{t} \beta_{i}+\int_{\Gamma}\left(u_{j} \partial_{j} u_{i}-h_{j} \partial_{j} h_{i}\right) d x^{\prime}=0
$$

or equivalently

$$
\beta_{i}(t)=\beta_{i}(0)-\int_{0}^{t} \int_{\Gamma}\left(u_{j} \partial_{j} u_{i}-h_{j} \partial_{j} h_{i}\right) d x^{\prime} d t
$$

Similarly, we have

$$
\gamma_{i}(t)=\gamma_{i}(0)-\int_{0}^{t} \int_{\Gamma}\left(u_{j} \partial_{j} h_{i}-h_{j} \partial_{j} u_{i}\right) d x^{\prime} d t .
$$

Finally, to solve $\hat{\mathbf{h}}$, and to recover $\mathbf{u}, \mathbf{h}$ from $\omega, \boldsymbol{\xi}$ in $\Omega_{f}$, one needs to solve two types of div-curl system. We leave it to the next section.

\section{Div-CURL SYSTEM}

In this section, we consider two div-curl systems, which have also been considered in [4] for the bounded domain. Assume that $\Gamma_{f}$ is a given graph with $f \in H^{s+\frac{1}{2}}\left(\mathbb{T}^{2}\right)$ for $s \geq 2$ satisfying

$$
-\left(1-c_{0}\right) \leq f \leq\left(1-c_{0}\right) .
$$

The first system reads as follows

$$
\left\{\begin{array}{l}
\operatorname{div} \mathbf{v}=g, \quad \operatorname{curl} \mathbf{v}=\omega \text { in } \Omega_{f} \\
\mathbf{v} \cdot \mathbf{N}_{f}=\vartheta \text { on } \Gamma_{f}, \mathbf{v} \cdot \mathbf{e}_{3}=0, \int_{\Gamma} v_{i} d x^{\prime}=\alpha_{i} \text { on } \Gamma, i=1,2,
\end{array}\right.
$$


where $\omega$ and $\vartheta$ are given functions in $\Omega_{f}$ and $\Gamma_{f}$ respectively, and $\alpha_{i}, i=1,2$ are given real numbers. Assume that $\omega, \theta$ satisfy the following compatibility conditions:

$$
\operatorname{div} \omega=0 \text { in } \Omega_{f}, \quad \int_{\Gamma} \omega_{3} d x^{\prime}=0, \quad \int_{\Omega_{f}} g d x=\int_{\mathbb{T}^{2}} \vartheta d x^{\prime} .
$$

The following proposition has been proved in [17].

Proposition 4.1. Let $f \in H^{s+\frac{1}{2}}, s \geq 2$ and $\sigma \in[1, s]$. Assume $g, \omega \in H^{\sigma-1}\left(\Omega_{f}\right)$, $\vartheta \in H^{\sigma-\frac{1}{2}}\left(\Gamma_{f}\right)$ satisfying (4.2). Then there exists a unique $\mathbf{v} \in H^{\sigma}\left(\Omega_{f}\right)$ of (4.1) so that

$$
\|\mathbf{v}\|_{H^{\sigma}\left(\Omega_{f}\right)} \leq C\left(\|f\|_{H^{s+\frac{1}{2}}}\right)\left(\|g\|_{H^{\sigma-1}\left(\Omega_{f}\right)}+\|\omega\|_{H^{\sigma-1}\left(\Omega_{f}\right)}+\|\vartheta\|_{H^{\sigma-\frac{1}{2}\left(\Gamma_{f}\right)}}+\left|\alpha_{1}\right|+\left|\alpha_{2}\right|\right) .
$$

The second div-curl system is

$$
\left\{\begin{array}{l}
\operatorname{div} \hat{\mathbf{h}}=\hat{g}, \quad \operatorname{curl} \hat{\mathbf{h}}=\hat{\boldsymbol{\omega}} \text { in } \Omega_{f}^{+}, \\
\hat{\mathbf{h}} \cdot \mathbf{N}_{f}=\hat{\vartheta} \text { on } \Gamma_{f}, \hat{\mathbf{h}} \times \mathbf{e}_{3}=\hat{\mathbf{J}} \text { on } \Gamma^{+} .
\end{array}\right.
$$

Here $\hat{\mathbf{J}}=\left(\hat{J}_{1}\left(x^{\prime}\right), \hat{J}_{2}\left(x^{\prime}\right), \hat{J}_{3}\left(x^{\prime}\right)\right)$ is a given vector on $\Gamma^{+}$. To solve this boundary value problem, we need the following compatibility conditions on $\hat{\boldsymbol{\omega}}$ and $\hat{\mathbf{J}}$ :

$$
\operatorname{div} \hat{\boldsymbol{\omega}}=0 \text { in } \Omega_{f}^{+}, \quad \partial_{1} \hat{J}_{1}+\partial_{2} \hat{J}_{2}=\hat{\omega}_{3}, \quad \hat{J}_{3}=0 \text { on } \Gamma^{+} .
$$

Proposition 4.2. Let $f \in H^{s+\frac{1}{2}}, s \geq 2$ and $\sigma \in[1, s]$. Assume $\hat{g}, \hat{\omega} \in H^{\sigma-1}\left(\Omega_{f}^{+}\right)$, $\hat{\vartheta}, \hat{\mathbf{J}} \in H^{\sigma-\frac{1}{2}}$ satisfying the compatibility condition (4.4). Then there exists a unique $\hat{\mathbf{h}} \in H^{\sigma}\left(\Omega_{f}^{+}\right)$of the div-curl system (4.3) so that

$$
\|\hat{\mathbf{h}}\|_{H^{\sigma}\left(\Omega_{f}^{+}\right)} \leq C\left(\|f\|_{H^{s+\frac{1}{2}}}\right)\left(\|\hat{g}\|_{H^{\sigma-1}\left(\Omega_{f}^{+}\right)}+\|\hat{\omega}\|_{H^{\sigma-1}\left(\Omega_{f}^{+}\right)}+\|\hat{\vartheta}\|_{H^{\sigma-\frac{1}{2}}}+\|\hat{\mathbf{J}}\|_{H^{\sigma-\frac{1}{2}}}\right) .
$$

Proof. By Proposition 4.1, there is a vector field $\tilde{\mathbf{h}}$ satisfies:

$$
\left\{\begin{array}{l}
\operatorname{div} \tilde{\mathbf{h}}=0, \quad \operatorname{curl} \tilde{\mathbf{h}}=\hat{\boldsymbol{\omega}} \text { in } \Omega_{f}^{+}, \\
\tilde{\mathbf{h}} \cdot \mathbf{N}_{f}=0 \text { on } \Gamma_{f}, \tilde{\mathbf{h}} \cdot \mathbf{e}_{3}=0 \text { on } \Gamma^{+},
\end{array}\right.
$$

and $\|\tilde{\mathbf{h}}\|_{H^{\sigma}\left(\Omega_{f}^{+}\right)} \leq C\left(\|f\|_{H^{s+\frac{1}{2}}}\right)\|\hat{\boldsymbol{\omega}}\|_{H^{\sigma-1}\left(\Omega_{f}^{+}\right)}$. On the other hand, according to (4.4), $\partial_{1}\left(\hat{J}_{1}-\right.$ $\left.\tilde{h}_{2}\right)+\partial_{2}\left(\hat{J}_{2}+\tilde{h}_{1}\right)=\partial_{1} \hat{J}_{1}+\partial_{2} \hat{J}_{2}-\hat{\omega}_{3}=0$, thus, there exists a scalar function $\hat{j} \in H^{\sigma+\frac{1}{2}}$ such that

$$
\hat{J}_{1}-\tilde{h}_{2}=\partial_{2} \hat{j}, \quad \hat{J}_{2}+\tilde{h}_{1}=-\partial_{1} \hat{j} .
$$

Let $\tilde{j} \in H^{\sigma+1}\left(\Omega_{f}^{+}\right)$be an extension of $\hat{j}$ to $\Omega_{f}^{+}$such that

$$
\tilde{j}=0 \text { near } \Gamma_{f}, \quad\|\tilde{j}\|_{H^{\sigma+1}\left(\Omega_{f}^{+}\right)} \leq C\left(\|f\|_{H^{s+\frac{1}{2}}}\right)\left(\|\hat{\omega}\|_{H^{\sigma-1}\left(\Omega_{f}^{+}\right)}+\|\hat{\mathbf{J}}\|_{H^{\sigma-\frac{1}{2}}}\right) .
$$

We consider the following mixed Dirichlet-Neumman problem:

$$
\left\{\begin{array}{l}
\Delta \phi=\Delta \tilde{j}-\hat{g} \quad \text { in } \Omega_{f}^{+}, \\
\nabla \phi \cdot \mathbf{N}_{f}=-\hat{\vartheta} \text { on } \Gamma_{f}, \phi=0 \text { on } \Gamma^{+} .
\end{array}\right.
$$

The existence, uniqueness and regularity of this mixed boundary value problem are standard [12]. Moreover,

$$
\|\phi\|_{H^{\sigma+1}\left(\Omega_{f}^{+}\right)} \leq C\left(\|f\|_{H^{s+\frac{1}{2}}}\right)\left(\|\hat{g}\|_{H^{\sigma-1}\left(\Omega_{f}^{+}\right)}+\|\hat{\boldsymbol{\omega}}\|_{H^{\sigma-1}\left(\Omega_{f}^{+}\right)}+\|\hat{\vartheta}\|_{H^{\sigma-\frac{1}{2}}}+\|\hat{\mathbf{J}}\|_{H^{\sigma-\frac{1}{2}}}\right) .
$$


Let

$$
\hat{\mathbf{h}}=\tilde{\mathbf{h}}+\nabla \tilde{j}-\nabla \phi .
$$

Obviously, $\operatorname{div} \hat{\mathbf{h}}=\Delta \tilde{j}-\Delta \phi=\hat{g}, \operatorname{curl} \hat{\mathbf{h}}=\operatorname{curl} \tilde{\mathbf{h}}=\hat{\boldsymbol{\omega}}$ in $\Omega_{f}^{+}$. Moreover,

$$
\hat{\mathbf{h}} \cdot \mathbf{N}_{f}=\tilde{\mathbf{h}} \cdot \mathbf{N}_{f}+\nabla \tilde{j} \cdot \mathbf{N}_{f}-\nabla \phi \cdot \mathbf{N}_{f}=\hat{\vartheta}
$$

due to $\tilde{j}=0$ near $\Gamma_{f}$. As to the boundary value on $\Gamma^{+}$, we note that since $\partial_{1} \phi, \partial_{2} \phi=0$ on $\Gamma^{+}$,

$\left.\hat{\mathbf{h}}\right|_{\Gamma^{+}} \times \mathbf{e}_{3}=\left.(\tilde{\mathbf{h}}+\nabla \tilde{j}-\nabla \phi)\right|_{\Gamma^{+}} \times \mathbf{e}_{3}=\left(\tilde{h}_{2}+\partial_{2} \hat{j}-\partial_{2} \phi,-\tilde{h}_{1}-\partial_{1} \hat{j}+\partial_{1} \phi, 0\right)=\left(\hat{J}_{1}, \hat{J}_{2}, 0\right)$.

We conclude the proof of existence of solution to the system (4.3) and the regularity estimate (4.5).

The proof of the uniqueness is similar to the proof of Lemma 5.4 in [17]. We present it here for completeness. It suffices to consider $\hat{g}, \hat{\boldsymbol{\omega}}, \hat{\vartheta}=0$ and $\hat{\mathbf{J}}=0$. We periodically extend $\Omega_{f}^{+}$to be a unbounded domain in $\mathbb{R}^{3}$, which is denoted by $\Omega_{f, p}^{+}$. Then $\hat{\mathbf{h}}=\nabla \phi$, where $\phi$ is a function on $\Omega_{f, p}^{+}$. Let $\zeta(x)=\phi\left(x_{1}+2 \pi, x_{2}, x_{3}\right)-\phi\left(x_{1}, x_{2}, x_{3}\right)$ for $x \in \Omega_{f, p}^{+}$. Then $\nabla \zeta(x)=0$, and thus $\zeta(x)$ is a constant. The condition $\partial_{1} \phi=\hat{h}_{1}=0$ on $\Gamma^{+}$implies $\zeta(x) \equiv 0$, and then $\phi$ is periodic in $x_{1}$ in $\Omega_{f, p}^{+}$. Similarly, $\phi$ is periodic in $x_{2}$. Thus $\phi$ can be viewed as a function on $\Omega_{f}^{+}$and is a constant on $\Gamma^{+}$. Then we can obtain a function $\phi$ in $\Omega_{f}^{+}$satisfies

$$
\Delta \phi=0 \quad \text { in } \Omega_{f}^{+}, \quad \mathbf{N}_{f} \cdot \nabla \phi=0 \quad \text { on } \Gamma_{f}, \quad \phi=\text { constant } \quad \text { on } \Gamma^{+} .
$$

Thus, the uniqueness of (4.6) implies $\phi \equiv$ constant, and then $\hat{\mathbf{h}} \equiv 0$.

\section{UNIFORM ESTIMATES FOR THE LINEARIZED SYSTEM}

Given $f\left(t, x^{\prime}\right), \mathbf{u}(t, x), \mathbf{h}(t, x), \hat{\mathbf{h}}(t, x)$, we assume there exist positive constants $\delta_{0}, c_{0}$ and $L_{0}, L_{1}, L_{2}$ such that for $t \in[0, T]$,

- $\|(\mathbf{u}, \mathbf{h}, \hat{\mathbf{h}})(t)\|_{L^{\infty}\left(\Gamma_{f}\right)} \leq L_{0}$

- $\|f(t)\|_{H^{s+\frac{1}{2}}}+\left\|\partial_{t} f(t)\right\|_{H^{s-\frac{1}{2}}}+\|(\mathbf{u}, \mathbf{h})(t)\|_{H^{s}\left(\Omega_{f}\right)}+\|\hat{\mathbf{h}}(t)\|_{H^{s}\left(\Omega_{f}^{+}\right)}+\left\|\partial_{t} \hat{\mathbf{h}}(t)\right\|_{H^{s-1}\left(\Omega_{f}^{+}\right)} \leq L_{1}$;

- $\|\left(\partial_{t} \mathbf{u}, \partial_{t} \mathbf{h} \|_{L^{\infty}\left(\Gamma_{f}\right)} \leq L_{2}\right.$;

- $\left\|f(t)-f_{*}\right\|_{H^{s-\frac{1}{2}}} \leq \delta_{0},-\left(1-c_{0}\right) \leq f\left(t, x^{\prime}\right) \leq\left(1-c_{0}\right), x^{\prime} \in \mathbb{T}^{2}$;

- $\Lambda(\mathbf{h}, \hat{\mathbf{h}})(t) \geq c_{1}$;

together with

$$
\left\{\begin{array} { l } 
{ \operatorname { d i v } \mathbf { u } = \operatorname { d i v } \mathbf { h } = 0 \text { in } \Omega _ { f } , } \\
{ \mathbf { h } \cdot \mathbf { N } _ { f } = 0 , \partial _ { t } f = \mathbf { u } \cdot \mathbf { N } _ { f } \text { on } \Gamma _ { f } , } \\
{ u _ { 3 } = h _ { 3 } = 0 \text { on } \Gamma , }
\end{array} \quad \left\{\begin{array}{l}
\operatorname{div} \hat{\mathbf{h}}=0, \operatorname{curl} \hat{\mathbf{h}}=0 \text { in } \Omega_{f}^{+}, \\
\hat{\mathbf{h}} \cdot \mathbf{N}_{f}=0 \text { on } \Gamma_{f}, \\
\hat{\mathbf{h}} \times \mathbf{e}_{3}=\hat{\mathbf{J}} \text { on } \Gamma^{+} .
\end{array}\right.\right.
$$

In this section, we linearize the equivalent system derived in Section 3 around $(f, \mathbf{u}, \mathbf{h}, \hat{\mathbf{h}})$, and present the uniform energy estimates for the linearized system. First of all, we give the following lemma on a new formulation of the stability condition (1.16).

Lemma 5.1. Under the stability condition (1.16), there exists $c_{1}>0$ such that

$$
\Lambda(\mathbf{h}, \hat{\mathbf{h}}) \stackrel{\text { def }}{=} \inf _{x \in \Gamma_{t}} \inf _{\varphi_{1}^{2}+\varphi_{2}^{2}=1}\left(h_{1} \varphi_{1}+h_{2} \varphi_{2}\right)^{2}+\left(\hat{h}_{1} \varphi_{1}+\hat{h}_{2} \varphi_{2}\right)^{2} \geq c_{1} .
$$


Proof. Let $\mathbf{q}=\left(q_{1}, q_{2}, q_{3}\right) \perp \mathbf{N}_{f}$ with $q_{3}=q_{1} \partial_{1} f+q_{2} \partial_{2} f$ and $\left(q_{1}, q_{2}\right)$ determined by

$$
\left(\begin{array}{cc}
1+\left(\partial_{1} f\right)^{2} & \partial_{1} f \partial_{2} f \\
\partial_{1} f \partial_{2} f & 1+\left(\partial_{2} f\right)^{2}
\end{array}\right)\left(\begin{array}{c}
q_{1} \\
q_{2}
\end{array}\right)=\left(\begin{array}{c}
\varphi_{1} \\
\varphi_{2}
\end{array}\right)
$$

Then by the fact $\mathbf{h} \cdot \mathbf{N}_{f}=\hat{\mathbf{h}} \cdot \mathbf{N}_{f}=0$, we get

$$
h_{1} \varphi_{1}+h_{2} \varphi_{2}=\sum_{i=1}^{3} h_{i} q_{i}, \hat{h}_{1} \varphi_{1}+\hat{h}_{2} \varphi_{2}=\sum_{i=1}^{3} \hat{h}_{i} q_{i}
$$

which along with (1.16) gives

$$
\inf _{\varphi_{1}^{2}+\varphi_{2}^{2}=1}\left(h_{1} \varphi_{1}+h_{2} \varphi_{2}\right)^{2}+\left(\hat{h}_{1} \varphi_{1}+\hat{h}_{2} \varphi_{2}\right)^{2}>0
$$

Since the inequality above holds for all $x \in \Gamma_{f}$, there exists a constant $c_{1}>0$ such that

$$
\inf _{\varphi_{1}^{2}+\varphi_{2}^{2}=1}\left(h_{1} \varphi_{1}+h_{2} \varphi_{2}\right)^{2}+\left(\hat{h}_{1} \varphi_{1}+\hat{h}_{2} \varphi_{2}\right)^{2} \geq c_{1}
$$

which yields (5.2).

For the system (3.2) and (3.11), we introduce the following linearized system:

$$
\left\{\begin{array}{l}
\partial_{t} \bar{f}=\bar{\theta}, \\
\partial_{t} \bar{\theta}=-2\left(\underline{u_{1}} \partial_{1} \bar{\theta}+\underline{u_{2}} \partial_{2} \bar{\theta}\right)+\sum_{i, j=1,2}\left(-\underline{u_{i}} \underline{u_{j}}+\underline{h_{i}} \underline{h_{j}}+\underline{\hat{h}_{i}} \underline{\hat{h}_{j}}\right) \partial_{i} \partial_{j} \bar{f}+\mathfrak{g},
\end{array}\right.
$$

where

$$
\mathfrak{g}=-\mathbf{N}_{f} \cdot \underline{\nabla\left(p_{\mathbf{u}, \mathbf{u}}-p_{\mathbf{h}, \mathbf{h}}\right)}-\frac{1}{2} \mathbf{N}_{f} \cdot \underline{\nabla\left(|\hat{\mathbf{h}}|^{2}-\widehat{\mathcal{H}}_{f}^{+}|\hat{\mathbf{h}}|^{2}\right)}+\frac{1}{2} \overline{\mathcal{N}}_{f}|\hat{\mathbf{h}}|^{2} .
$$

We remark that $\int_{\mathbb{T}^{2}} \bar{\theta} d x^{\prime}$ may not vanish since we have performed a linearization.

Now we introduce the energy functional $E_{s}$ defined by

$$
E_{s}(t)=\left\|\left(\partial_{t}+\underline{u_{i}} \partial_{i}\right)\langle\nabla\rangle^{s-\frac{1}{2}} \bar{f}\right\|_{L^{2}}^{2}+\frac{1}{2}\left\|\underline{h_{i}} \partial_{i}\langle\nabla\rangle^{s-\frac{1}{2}} \bar{f}\right\|_{L^{2}}^{2}+\frac{1}{2}\left\|\hat{h}_{i} \partial_{i}\langle\nabla\rangle^{s-\frac{1}{2}} \bar{f}\right\|_{L^{2}}^{2} .
$$

Also we define the standard energy

$$
\mathcal{E}_{s}(t)=\|\bar{f}(t)\|_{H^{s+\frac{1}{2}}}^{2}+\left\|\partial_{t} \bar{f}(t)\right\|_{H^{s-\frac{1}{2}}}^{2} .
$$

It is easy to see that there exists $C\left(L_{0}\right)>0$ so that

$$
E_{s}(t) \leq C\left(L_{0}\right) \mathcal{E}_{s}(t) .
$$

The stability condition guarantees that there exists $C\left(c_{1}, L_{0}\right)$ so that

$$
\mathcal{E}_{s}(t) \leq C\left(c_{0}, L_{0}\right)\left\{E_{s}(t)+\left\|\partial_{t} \bar{f}\right\|_{L^{2}}^{2}+\|\bar{f}\|_{L^{2}}^{2}\right\} .
$$

Before to state the energy estimates, we first give the following lemma concerning $\mathfrak{g}$ defined by (5.4).

Lemma 5.2. It holds that

$$
\|\mathfrak{g}\|_{H^{s-\frac{1}{2}}} \leq C\left(L_{1}\right)
$$


Proof. According to the definition of $p_{\mathbf{u}, \mathbf{u}}, p_{\mathbf{h}, \mathbf{h}}$ (see (3.6) ), we obtain by standard elliptic estimates that

$$
\begin{aligned}
\| \mathbf{N}_{f} \cdot \underline{\nabla\left(p_{\mathbf{u}, \mathbf{u}}-p_{\mathbf{h}, \mathbf{h}}\right) \|_{H^{s-\frac{1}{2}}}} & \leq C\left(L_{1}\right)\left\|\nabla\left(p_{\mathbf{u}, \mathbf{u}}-p_{\mathbf{h}, \mathbf{h}}\right)\right\|_{H^{s-\frac{1}{2}}} \leq C\left(L_{1}\right)\left\|\nabla\left(p_{\mathbf{u}, \mathbf{u}}, p_{\mathbf{h}, \mathbf{h}}\right)\right\|_{H^{s}\left(\Omega_{f}\right)} \\
& \leq C\left(L_{1}\right)\|(\mathbf{u}, \mathbf{h})\|_{H^{s}\left(\Omega_{f}\right)} \leq C\left(L_{1}\right) .
\end{aligned}
$$

Applying similar argument to $\hat{p}=|\hat{\mathbf{h}}|^{2}-\widehat{\mathcal{H}}_{f}^{+}|\hat{\mathbf{h}}|^{2}$ yields the same estimate for the second term in $\mathfrak{g}$. Finally, the same estimate for the third term follows from (2.7) in Proposition 2.1.

Proposition 5.1. Given initial data $\bar{f}_{0} \in H^{s+\frac{1}{2}}, \bar{\theta}_{0} \in H^{s-\frac{1}{2}}$, there exists a unique solution $(\bar{f}, \bar{\theta}) \in C\left([0, T] ; H^{s+\frac{1}{2}} \times H^{s-\frac{1}{2}}\right)$ to the system (5.3) so that

$$
\sup _{t \in[0, T]} \mathcal{E}_{s}(t) \leq C\left(c_{1}, L_{0}\right)\left(1+\left\|\bar{\theta}_{0}\right\|_{H^{s-\frac{1}{2}}}^{2}+\left\|\bar{f}_{0}\right\|_{H^{s+\frac{1}{2}}}^{2}\right) e^{C\left(c_{1}, L_{1}, L_{2}\right) T} .
$$

Proof. We only present the uniform estimates, which ensure the existence and uniqueness of the solution. Using the fact that

$$
\partial_{t}^{2} \bar{f}=-2 \sum_{i=1,2} \underline{u_{i}} \partial_{i} \partial_{t} \bar{f}+\sum_{i, j=1,2}\left(-\underline{u_{i}} \underline{u_{j}}+\underline{h_{i}} \underline{h_{j}}+\underline{\hat{h}_{i}} \underline{\hat{h}_{j}}\right) \partial_{i} \partial_{j} \bar{f}+\mathfrak{g},
$$

a direct calculation shows that

$$
\begin{aligned}
\frac{1}{2} & \frac{d}{d t}\left\|\left(\partial_{t}+\underline{u_{i}} \partial_{i}\right)\langle\nabla\rangle^{s-\frac{1}{2}} \bar{f}\right\|_{L^{2}\left(\mathbb{T}^{2}\right)}^{2} \\
= & \left\langle\left(\partial_{t}+\underline{u_{i}} \partial_{i}\right)\langle\nabla\rangle^{s-\frac{1}{2}} \bar{f},\langle\nabla\rangle^{s-\frac{1}{2}} \partial_{t}^{2} \bar{f}+\underline{u_{i}} \partial_{i}\left(\langle\nabla\rangle^{s-\frac{1}{2}} \partial_{t} \bar{f}\right)+\partial_{t} \underline{u_{i}} \partial_{i}\langle\nabla\rangle^{s-\frac{1}{2}} \bar{f}\right\rangle \\
= & \left.\left\langle\left(\partial_{t}+\underline{u_{i}} \partial_{i}\right)\langle\nabla\rangle^{s-\frac{1}{2}} \bar{f},\langle\nabla\rangle^{s-\frac{1}{2}}\left(-2 \underline{u_{i}} \partial_{i} \partial_{t} \bar{f}-\underline{u_{i}} \underline{u_{j}} \partial_{i} \partial_{j} \bar{f}+\underline{\left(h_{i}\right.} \underline{h_{j}}+\underline{\hat{h}_{i}} \underline{h_{j}}\right) \partial_{i} \partial_{j} \bar{f}\right)\right\rangle \\
+ & \left\langle\left(\partial_{t}+\underline{u_{i}} \partial_{i}\right)\langle\nabla\rangle^{s-\frac{1}{2}} \bar{f},\langle\nabla\rangle^{s-\frac{1}{2}} \mathfrak{g}+\underline{u_{i}} \partial_{i}\left(\langle\nabla\rangle^{s-\frac{1}{2}} \partial_{t} \bar{f}\right)+\partial_{t} \underline{u_{i}} \partial_{i}\langle\nabla\rangle^{s-\frac{1}{2}} \bar{f}\right\rangle \\
= & \left\langle\left(\partial_{t}+\underline{u_{i}} \partial_{i}\right)\langle\nabla\rangle^{s-\frac{1}{2}} \bar{f},-\underline{u_{i}} \partial_{i}\langle\nabla\rangle^{s-\frac{1}{2}} \partial_{t} \bar{f}\right\rangle \\
& \left.\left.+\left\langle\left(\partial_{t}+\underline{u_{i}} \partial_{i}\right)\langle\nabla\rangle^{s-\frac{1}{2}} \bar{f},-\underline{u_{i}} \underline{u_{j}} \partial_{i} \partial_{j}\langle\nabla\rangle^{s-\frac{1}{2}} \bar{f}+\underline{\left(h_{i}\right.} \underline{h_{j}}+\underline{h_{i}} \underline{h_{j}}\right) \partial_{i} \partial_{j}\langle\nabla\rangle^{s-\frac{1}{2}} \bar{f}\right)\right\rangle \\
& \left.+2\left\langle\left(\partial_{t}+\underline{u_{i}} \partial_{i}\right)\langle\nabla\rangle^{s-\frac{1}{2}} \bar{f}, \underline{\left[u_{i}\right.},\langle\nabla\rangle^{s-\frac{1}{2}}\right] \partial_{i} \partial_{t} \bar{f}\right\rangle \\
& \left.\left.+\left\langle\left(\partial_{t}+\underline{u_{i}} \partial_{i}\right)\langle\nabla\rangle^{s-\frac{1}{2}} \bar{f}, \underline{u_{i}} \underline{u_{j}}-\underline{h_{i}} \underline{h_{j}}-\underline{\hat{h}_{i}} \underline{\hat{h}_{j}},\langle\nabla\rangle^{s-\frac{1}{2}}\right] \partial_{i} \partial_{j} \bar{f}\right)\right\rangle \\
& +\left\langle\left(\partial_{t}+\underline{u_{i}} \partial_{i}\right)\langle\nabla\rangle^{s-\frac{1}{2}} \bar{f},\langle\nabla\rangle^{s-\frac{1}{2}} \mathfrak{g}+\partial_{t} \underline{u_{i}} \partial_{i}\langle\nabla\rangle^{s-\frac{1}{2}} \bar{f}\right\rangle \triangleq I_{1}+\cdots+I_{5} .
\end{aligned}
$$

It follows from Lemma 2.2 that

$$
\begin{aligned}
I_{3} & \leq 2\left\|\left(\partial_{t}+\underline{u_{i}} \partial_{i}\right)\langle\nabla\rangle^{s-\frac{1}{2}} \bar{f}\right\|_{L^{2}}\left\|\left[\underline{u_{i}},\langle\nabla\rangle^{s-\frac{1}{2}}\right] \partial_{i} \partial_{t} \bar{f}\right\|_{L^{2}} \\
& \leq C E_{s}(t)^{\frac{1}{2}}\|\underline{\mathbf{u}}\|_{H^{s-\frac{1}{2}}}\left\|\partial_{t} \bar{f}\right\|_{H^{s-\frac{1}{2}}},
\end{aligned}
$$

as well as

$$
I_{4} \leq C E_{s}(t)^{\frac{1}{2}}\left(\left\|\underline{u}_{H^{s-\frac{1}{2}}}^{2}+\right\| \underline{\mathbf{h}}_{H^{s-\frac{1}{2}}}^{2}+\|\underline{\hat{\mathbf{h}}}\|_{H^{s-\frac{1}{2}}}^{2}\right)\|\bar{f}\|_{H^{s+\frac{1}{2}}} \cdot
$$

Also we have

$$
I_{5} \leq E_{s}(t)^{\frac{1}{2}}\left(\|\mathfrak{g}\|_{H^{s-\frac{1}{2}}}+\left\|\partial_{t} \mathbf{u}\right\|_{L^{\infty}}\|\bar{f}\|_{H^{s+\frac{1}{2}}}\right)
$$


We get by integration by parts that

$$
\begin{aligned}
& \left\langle\partial_{t}\langle\nabla\rangle^{s-\frac{1}{2}} \bar{f},-\underline{u_{i}} \partial_{i}\langle\nabla\rangle^{s-\frac{1}{2}} \partial_{t} \bar{f}\right\rangle \leq\left\|\partial_{i} \underline{u_{i}}\right\|_{L^{\infty}}\left\|\partial_{t} \bar{f}\right\|_{H^{s-\frac{1}{2}}}^{2}, \\
& \left\langle\underline{u_{i}} \partial_{i}\langle\nabla\rangle^{s-\frac{1}{2}} \bar{f},-\underline{u_{i}} \partial_{i}\langle\nabla\rangle^{s-\frac{1}{2}} \partial_{t} \bar{f}\right\rangle+\frac{1}{2} \frac{d}{d t}\left\|\underline{u}_{i} \partial_{i}\langle\nabla\rangle^{s-\frac{1}{2}} \bar{f}\right\|_{L^{2}}^{2} \\
& =\left\langle\underline{u_{i}} \partial_{i}\langle\nabla\rangle^{s-\frac{1}{2}} \bar{f}, \partial_{t} \underline{u}_{i} \partial_{i}\langle\nabla\rangle^{s-\frac{1}{2}} \bar{f}\right\rangle \leq\|\mathbf{u}\|_{L^{\infty}}\left\|\partial_{t} \mathbf{u}\right\|_{L^{\infty}}\|\bar{f}\|_{H^{s+\frac{1}{2}}}^{2},
\end{aligned}
$$

which give rise to

$$
I_{1} \leq-\frac{1}{2} \frac{d}{d t}\left\|\underline{u}_{i} \partial_{i}\langle\nabla\rangle^{s-\frac{1}{2}} \bar{f}\right\|_{L^{2}}^{2}+\left(1+\|\mathbf{u}\|_{W^{1, \infty}}+\left\|\partial_{t} \mathbf{u}\right\|_{L^{\infty}}\right)^{2}\left(\|\bar{f}\|_{H^{s+\frac{1}{2}}}^{2}+\left\|\partial_{t} \bar{f}\right\|_{H^{s-\frac{1}{2}}}^{2}\right) .
$$

Similarly, we have

$$
\begin{aligned}
& \left\langle\partial_{t}\langle\nabla\rangle^{s-\frac{1}{2}} \bar{f},-\underline{u_{i}} \underline{u}_{j} \partial_{i} \partial_{j}\langle\nabla\rangle^{s-\frac{1}{2}} \bar{f}\right\rangle-\frac{1}{2} \frac{d}{d t}\left\|\underline{u_{i}} \partial_{i}\langle\nabla\rangle^{s-\frac{1}{2}} \bar{f}\right\|_{L^{2}}^{2} \\
& =-\left\langle\underline{u_{i}} \partial_{i}\langle\nabla\rangle^{s-\frac{1}{2}} \bar{f}, \partial_{t} \underline{u_{i}} \partial_{i}\langle\nabla\rangle^{s-\frac{1}{2}} \bar{f}\right\rangle+\left\langle\langle\nabla\rangle^{s-\frac{1}{2}} \partial_{t} \bar{f}, \partial_{i}\left(\underline{u_{i}} \underline{u}_{j}\right) \partial_{j}\langle\nabla\rangle^{s-\frac{1}{2}} \bar{f}\right\rangle \\
& \leq\|\mathbf{u}\|_{L^{\infty}}\left(\left\|\partial_{t} \mathbf{u}\right\|_{L^{\infty}}+\|\nabla \mathbf{u}\|_{L^{\infty}}\right)\left(\|\bar{f}\|_{H^{s+\frac{1}{2}}}^{2}+\left\|\partial_{t} \bar{f}\right\|_{H^{s-\frac{1}{2}}}^{2}\right),
\end{aligned}
$$

and

$$
\begin{aligned}
& \left\langle\underline{u_{k}} \partial_{k}\langle\nabla\rangle^{s-\frac{1}{2}} \bar{f},-\underline{u_{i}} \underline{u_{j}} \partial_{i} \partial_{j}\langle\nabla\rangle^{s-\frac{1}{2}} \bar{f}\right\rangle \\
& =\left\langle\partial_{i}\left(\underline{u_{k} u_{i}} \underline{u_{j}}\right) \partial_{k}\langle\nabla\rangle^{s-\frac{1}{2}} \bar{f}, \partial_{j}\langle\nabla\rangle^{s-\frac{1}{2}} \bar{f}\right\rangle-\left\langle\underline{u_{k} u_{i} u_{j}} \partial_{k} \partial_{i}\langle\nabla\rangle^{s-\frac{1}{2}} \bar{f}, \partial_{j}\langle\nabla\rangle^{s-\frac{1}{2}} \bar{f}\right\rangle \\
& =\left\langle\partial_{i}\left(\underline{u_{k} u_{i}} \underline{u_{j}}\right) \partial_{k}\langle\nabla\rangle^{s-\frac{1}{2}} \bar{f}, \partial_{j}\langle\nabla\rangle^{s-\frac{1}{2}} \bar{f}\right\rangle-\left\langle\underline{u_{k}} \partial_{k}\left(\underline{u_{i}} \partial_{i}\langle\nabla\rangle^{s-\frac{1}{2}} \bar{f}\right), \underline{u}_{j} \partial_{j}\langle\nabla\rangle^{s-\frac{1}{2}} \bar{f}\right\rangle \\
& \left.\quad+\left\langle\underline{u_{k}}\left(\partial_{k} \underline{u_{i}}\right) \partial_{i}\langle\nabla\rangle^{s-\frac{1}{2}} \bar{f}\right), \underline{u_{j}} \partial_{j}\langle\nabla\rangle^{s-\frac{1}{2}} \bar{f}\right\rangle \leq C\|\mathbf{u}\|_{L^{\infty}}^{2}\|\nabla \mathbf{u}\|_{L^{\infty}}\|\bar{f}\|_{H^{s+\frac{1}{2}}}^{2},
\end{aligned}
$$

as well as

$$
\begin{aligned}
& \left\langle\partial_{t}\langle\nabla\rangle^{s-\frac{1}{2}} \bar{f}, \underline{h_{i}} \underline{h_{j}} \partial_{i} \partial_{j}\langle\nabla\rangle^{s-\frac{1}{2}} \bar{f}\right\rangle \\
& \leq-\frac{1}{2} \frac{d}{d t}\left\|\underline{h_{i}} \partial_{i}\langle\nabla\rangle^{s-\frac{1}{2}} \bar{f}\right\|_{L^{2}}^{2}+\|\mathbf{h}\|_{L^{\infty}}\left(\left\|\partial_{t} \mathbf{h}\right\|_{L^{\infty}}+\|\nabla \mathbf{h}\|_{L^{\infty}}\right)\left(\|\bar{f}\|_{H^{s+\frac{1}{2}}}^{2}+\left\|\partial_{t} \bar{f}\right\|_{H^{s-\frac{1}{2}}}^{2}\right), \\
& \left\langle\partial_{t}\langle\nabla\rangle^{s-\frac{1}{2}} \bar{f}, \underline{\hat{h}_{i}} \underline{\hat{h}_{j}} \partial_{i} \partial_{j}\langle\nabla\rangle^{s-\frac{1}{2}} \bar{f}\right\rangle \\
& \leq-\frac{1}{2} \frac{d}{d t}\left\|\hat{h}_{i} \partial_{i}\langle\nabla\rangle^{s-\frac{1}{2}} \bar{f}\right\|_{L^{2}}^{2}+\|\hat{\mathbf{h}}\|_{L^{\infty}}\left(\left\|\partial_{t} \hat{\mathbf{h}}\right\|_{L^{\infty}}+\|\nabla \hat{\mathbf{h}}\|_{L^{\infty}}\right)\left(\|\bar{f}\|_{H^{s+\frac{1}{2}}}^{2}+\left\|\partial_{t} \bar{f}\right\|_{H^{s-\frac{1}{2}}}^{2}\right) .
\end{aligned}
$$

Thus, we obtain

$$
\begin{aligned}
I_{2} \leq & \frac{1}{2} \frac{d}{d t}\left\|\underline{u_{i}} \partial_{i}\langle\nabla\rangle^{s-\frac{1}{2}} \bar{f}\right\|_{L^{2}}^{2}-\frac{1}{4} \frac{d}{d t}\left\|\underline{h_{i}} \partial_{i}\langle\nabla\rangle^{s-\frac{1}{2}} \bar{f}\right\|_{L^{2}}^{2}-\frac{1}{4} \frac{d}{d t}\left\|\hat{h}_{i} \partial_{i}\langle\nabla\rangle^{s-\frac{1}{2}} \bar{f}\right\|_{L^{2}}^{2} \\
& +C\left(1+\|(\mathbf{u}, \mathbf{h}, \hat{\mathbf{h}})\|_{W^{1, \infty}}+\left\|\left(\partial_{t} \mathbf{u}, \partial_{t} \mathbf{h}, \partial_{t} \hat{\mathbf{h}}\right)\right\|_{L^{\infty}}\right)^{3}\left(\|\bar{f}\|_{H^{s+\frac{1}{2}}}^{2}+\left\|\partial_{t} \bar{f}\right\|_{H^{s-\frac{1}{2}}}^{2}\right) .
\end{aligned}
$$

Collecting these estimates of $I_{1}, \cdots, I_{5}$ above, we conclude that

$$
\frac{d}{d t} E_{s}(t) \leq\|\mathfrak{g}\|_{H^{s-\frac{1}{2}}}^{2}+C\left(L_{0}\right)\left(1+\|(\underline{\mathbf{u}}, \underline{\mathbf{h}}, \underline{\hat{\mathbf{h}}})\|_{H^{s-\frac{1}{2}}}+\left\|\left(\partial_{t} \mathbf{u}, \partial_{t} \mathbf{h}, \partial_{t} \hat{\mathbf{h}}\right)\right\|_{L^{\infty}}\right)^{3} \mathcal{E}_{s}(t) .
$$


On the other hand, it is obvious that

$$
\frac{d}{d t}\left(\left\|\partial_{t} \bar{f}\right\|_{L^{2}}^{2}+\|\bar{f}\|_{L^{2}}^{2}\right) \leq C\left(L_{0}\right) \mathcal{E}_{s}(t)+\|\mathfrak{g}\|_{L^{2}}^{2} .
$$

Then by (5.6), we deduce that

$$
\begin{aligned}
\mathcal{E}_{s}(t) \leq & C\left(c_{1}, L_{0}\right)\left(\left\|\bar{\theta}_{0}\right\|_{H^{s-\frac{1}{2}}}^{2}+\left\|\bar{f}_{0}\right\|_{H^{s+\frac{1}{2}}}^{2}+\int_{0}^{t}\|\mathfrak{g}(\tau)\|_{H^{s-\frac{1}{2}}}^{2} d \tau\right. \\
& \left.+\int_{0}^{t}\left(1+\|(\underline{\mathbf{u}}, \underline{\mathbf{h}}, \underline{\hat{\mathbf{h}}})(\tau)\|_{H^{s-\frac{1}{2}}}+\left\|\partial_{t}(\mathbf{u}, \mathbf{h}, \hat{\mathbf{h}})(\tau)\right\|_{L^{\infty}}\right)^{3} \mathcal{E}_{s}(\tau) d \tau\right),
\end{aligned}
$$

which together with Lemma 2.1 gives rise to

$$
\mathcal{E}_{s}(t) \leq C\left(c_{1}, L_{0}\right)\left(\left\|\bar{\theta}_{0}\right\|_{H^{s-\frac{1}{2}}}^{2}+\left\|\bar{f}_{0}\right\|_{H^{s+\frac{1}{2}}}^{2}+\int_{0}^{t}\|\mathfrak{g}(\tau)\|_{H^{s-\frac{1}{2}}}^{2} d \tau+C\left(L_{1}, L_{2}\right) \int_{0}^{t} \mathcal{E}_{s}(\tau) d \tau\right) .
$$

The desired estimate (5.7) follows from Lemma 5.2 and Gronwall's inequality.

For the vorticity and current system (3.12)-(3.13), we introduce the following linearized system:

$$
\begin{aligned}
& \partial_{t} \overline{\boldsymbol{\omega}}+\mathbf{u} \cdot \nabla \overline{\boldsymbol{\omega}}-\mathbf{h} \cdot \nabla \overline{\boldsymbol{\xi}}=\overline{\boldsymbol{\omega}} \cdot \nabla \mathbf{u}-\overline{\boldsymbol{\xi}} \cdot \nabla \mathbf{h} \text { in } Q_{T}, \\
& \partial_{t} \overline{\boldsymbol{\xi}}+\mathbf{u} \cdot \nabla \overline{\boldsymbol{\xi}}-\mathbf{h} \cdot \nabla \overline{\boldsymbol{\omega}}=\overline{\boldsymbol{\xi}} \cdot \nabla \mathbf{u}-\overline{\boldsymbol{\omega}} \cdot \nabla \mathbf{h}-2 \nabla u_{i} \times \nabla h_{i} \text { in } Q_{T},
\end{aligned}
$$

together with the initial data

$$
\overline{\boldsymbol{\omega}}(0, x)=\overline{\boldsymbol{\omega}}_{0}(x), \quad \overline{\boldsymbol{\xi}}(0, x)=\overline{\boldsymbol{\xi}}_{0}(x), x \in \Omega_{f} .
$$

The following proposition can be proved in a standard way(see [17]).

Proposition 5.2. Given $\bar{\omega}_{0}, \overline{\boldsymbol{\xi}}_{0} \in H^{s-1}\left(\Omega_{f}\right)$, there exists a unique solution $(\overline{\boldsymbol{\omega}}, \overline{\boldsymbol{\xi}})$ to the initial value problem (5.8)-(5.10) such that

$$
\sup _{t \in[0, T]}\left(\|\overline{\boldsymbol{\omega}}(t)\|_{H^{s-1}\left(\Omega_{f}\right)}^{2}+\|\overline{\boldsymbol{\xi}}(t)\|_{H^{s-1}\left(\Omega_{f}\right)}^{2}\right) \leq\left(1+\left\|\overline{\boldsymbol{\omega}}_{0}\right\|_{H^{s-1}\left(\Omega_{0}\right)}^{2}+\left\|\overline{\boldsymbol{\xi}}_{0}\right\|_{H^{s-1}\left(\Omega_{0}\right)}^{2}\right) e^{C\left(L_{1}\right) T} .
$$

Moreover, it holds that

$$
\frac{d}{d t} \int_{\Gamma} \bar{\omega}_{3} d x^{\prime}=0, \quad \frac{d}{d t} \int_{\Gamma} \bar{\xi}_{3} d x^{\prime}=0
$$

Finally, the magnetic field $\hat{\mathbf{h}}$ in the vacuum is considered as a secondary variable computed from $\Gamma_{f}$ and $\hat{\mathbf{J}}$ by solving the following div-curl system:

$$
\left\{\begin{array}{l}
\operatorname{curl} \hat{\mathbf{h}}=0, \operatorname{div} \hat{\mathbf{h}}=0 \text { in } \Omega_{f}^{+}, \\
\hat{\mathbf{h}} \cdot \mathbf{N}_{f}=0 \text { on } \Gamma_{f}, \hat{\mathbf{h}} \times \mathbf{e}_{3}=\hat{\mathbf{J}} \text { on } \Gamma^{+},
\end{array}\right.
$$

for any fixed time $t \geq 0$. According to Proposition 4.2

$$
\|\hat{\mathbf{h}}(t)\|_{H^{s}\left(\Omega_{f}^{+}\right)} \leq C\left(\|f(t)\|_{H^{s+\frac{1}{2}}}\right)\|\hat{\mathbf{J}}(t)\|_{H^{s-\frac{1}{2}}} .
$$




\section{Construction of THE iteration maP}

Given $f_{0} \in H^{s+\frac{1}{2}}\left(\mathbb{T}^{2}\right), \mathbf{u}_{0}, \mathbf{h}_{0} \in H^{s}\left(\Omega_{f_{0}}\right)$ and $\hat{\mathbf{J}} \in C^{k}\left(\left[0, T_{0}\right] ; H^{s-\frac{1}{2}-k}\right), k=0$, we first solve $\hat{\mathbf{h}}_{0} \in H^{s}\left(\Omega_{f_{0}}^{+}\right)$from (1.13) as mentioned before, and assume furthermore that there exists $c_{0}, c_{1}>0$ so that

$$
-1+\frac{c_{0}}{2} \leq f_{0}\left(x^{\prime}\right) \leq 1-\frac{c_{0}}{2}, \quad \Lambda\left(\mathbf{h}_{0}, \hat{\mathbf{h}}_{0}\right) \geq 2 c_{1} .
$$

We then choose $f_{*}=f_{0}$ and take $\Omega_{*}=\Omega_{f_{0}}$ as the reference region. The initial data $\left(f_{I},\left(\partial_{t} f\right)_{I}, \omega_{* I}, \boldsymbol{\xi}_{* I}, \beta_{I i}, \gamma_{I i}\right)$ for the equivalent system formulated at the beginning of Section 3 is defined as follows:

$$
\begin{aligned}
& f_{I}=f_{0},\left(\partial_{t} f\right)_{I}=\mathbf{u}_{0}\left(x^{\prime}, f_{0}\left(x^{\prime}\right)\right) \cdot\left(-\partial_{1} f_{0},-\partial_{2} f_{0}, 1\right), \\
& \omega_{* I}=\operatorname{curl} \mathbf{u}_{0}, \boldsymbol{\xi}_{* I}=\operatorname{curl} \mathbf{h}_{0}, \\
& \beta_{I i}=\int_{\mathbb{T}^{2}} u_{0 i}\left(x^{\prime},-1\right) d x^{\prime}, \gamma_{I i}=\int_{\mathbb{T}^{2}} h_{0 i}\left(x^{\prime},-1\right) d x^{\prime} .
\end{aligned}
$$

In addition, we choose a large constant $M_{0}>1$ so that

$$
\begin{aligned}
& \left\|f_{I}\right\|_{H^{s+\frac{1}{2}}}+\left\|\left(\omega_{I *}, \boldsymbol{\xi}_{I *}\right)\right\|_{H^{s-1}\left(\Omega_{*}\right)}+\left\|\left(\partial_{t} f\right)_{I}\right\|_{H^{s-\frac{1}{2}}}+\left|\beta_{I i}\right|+\left|\gamma_{I i}\right|+\left\|\hat{\mathbf{h}}_{0}\right\|_{H^{s}\left(\Omega_{*}\right)} \\
& +\|\hat{\mathbf{J}}(t)\|_{H^{s-\frac{1}{2}\left(\Gamma^{+}\right)}}+\left\|\partial_{t} \hat{\mathbf{J}}(t)\right\|_{H^{s-\frac{3}{2}\left(\Gamma^{+}\right)}} \leq M_{0} .
\end{aligned}
$$

The iteration map we constructed is essentially based on iterating the unknowns $f, \omega_{*}, \boldsymbol{\xi}_{*}, \beta_{i}, \gamma_{i}$, which satisfy certain evolution equations, while the secondary variable $\hat{\mathbf{h}}$ is determined by $(f, \hat{\mathbf{J}})$ through (5.11).

Now we introduce the following functional space.

Definition 6.1. Given two positive constants $M_{1}, M_{2}>0$ with $M_{1} \geq 2 M_{0}$, we define the space $\mathcal{X}=\mathcal{X}\left(T, M_{1}, M_{2}\right)$ as the collection of $\left(f, \omega_{*}, \boldsymbol{\xi}_{*}, \beta_{i}, \gamma_{i}\right)$, which satisfies

$$
\begin{gathered}
\left(f(0), \partial_{t} f(0), \omega_{*}(0), \boldsymbol{\xi}_{*}(0), \beta_{i}(0), \gamma_{i}(0)\right)=\left(f_{I},\left(\partial_{t} f\right)_{I}, \omega_{* I}, \boldsymbol{\xi}_{* I}, \beta_{i I}, \gamma_{i I}\right), \\
\sup _{t \in[0, T]}\left\|f(t, \cdot)-f_{*}\right\|_{H^{s-\frac{1}{2}}} \leq \delta_{0},
\end{gathered}
$$

$$
\sup _{t \in[0, T]}\left(\left\|\partial_{t}^{2} f(t)\right\|_{H^{s-\frac{3}{2}}}+\left\|\left(\partial_{t} \omega_{*}, \partial_{t} \xi_{*}\right)(t)\right\|_{H^{s-2}\left(\Omega_{*}\right)}+\left|\partial_{t} \beta_{i}(t)\right|+\left|\partial_{t} \gamma_{i}(t)\right|\right) \leq M_{2}
$$

together with the following compatibility conditions:

$$
\int_{\mathbb{T}^{2}} \partial_{t} f\left(t, x^{\prime}\right) d x^{\prime}=0, \int_{\Gamma} \omega_{* 3} d x^{\prime}=\int_{\Gamma} \boldsymbol{\xi}_{* 3} d x^{\prime}=0
$$

Given $\left(f, \omega_{*}, \boldsymbol{\xi}_{*}, \beta_{i}, \gamma_{i}\right) \in \mathcal{X}\left(T, M_{1}, M_{2}\right)$, our goal is to construct an iteration map

$$
\left(\bar{f}, \overline{\boldsymbol{\omega}}_{*}, \overline{\boldsymbol{\xi}}_{*}, \bar{\beta}_{i}, \bar{\gamma}_{i}\right)=\mathcal{F}\left(f, \omega_{*}, \boldsymbol{\xi}_{*}, \beta_{i}, \gamma_{i}\right) \in \mathcal{X}\left(T, M_{1}, M_{2}\right)
$$

with suitably chosen constants $M_{1}, M_{2}$ and $T$. 
6.1. Recover the bulk region, velocity and magnetic fields. Recall that

$$
\Omega_{f}^{+}=\left\{x \in \Omega \mid x_{3}>f\left(t, x^{\prime}\right)\right\}, \Omega_{f}\left(=\Omega_{f}^{-}\right)=\left\{x \in \Omega \mid x_{3}<f\left(t, x^{\prime}\right)\right\},
$$

and the harmonic coordinate map $\Phi_{f}: \Omega_{*} \rightarrow \Omega_{f}$ defined in (2.6).

We first define $\hat{\mathbf{h}}$ by solving (5.11). Then $\partial_{t} \hat{\mathbf{h}}$ satisfies

$$
\left\{\begin{array}{l}
\operatorname{curl} \partial_{t} \hat{\mathbf{h}}=0, \operatorname{div} \partial_{t} \hat{\mathbf{h}}=0 \text { in } \Omega_{f}^{+}, \\
\partial_{t} \hat{\mathbf{h}} \cdot \mathbf{N}_{f}=-\partial_{t} f \partial_{3} \hat{\mathbf{h}} \cdot \mathbf{N}_{f}+\hat{h}_{1} \partial_{1} \partial_{t} f+\hat{h}_{2} \partial_{2} \partial_{t} f \text { on } \Gamma_{f}, \\
\partial_{t} \hat{\mathbf{h}} \times \mathbf{e}_{3}=\partial_{t} \hat{\mathbf{J}} \text { on } \Gamma^{+} .
\end{array}\right.
$$

It follows from Proposition 4.2 that

$$
\|\hat{\mathbf{h}}(t)\|_{H^{s}\left(\Omega_{f}^{+}\right)}+\left\|\partial_{t} \hat{\mathbf{h}}(t)\right\|_{H^{s-1}\left(\Omega_{f}^{+}\right)} \leq C\left(M_{0}, M_{1}\right) .
$$

To recover $\mathbf{u}, \mathbf{h}$ in $\Omega_{f}$, we define an operator which projects any vector field in $\Omega_{f}$ to its divergence-free part. More precisely, for any $\omega \in H^{s}\left(\Omega_{f}\right)$, let $P_{f}^{\text {div }} \omega=\omega-\nabla \phi$ with $\phi$ solving the following mixed boundary value problem:

$$
\Delta \phi=\operatorname{div} \omega \text { in } \Omega_{f}, \partial_{3} \phi=0 \text { on } \Gamma, \phi=0 \text { on } \Gamma_{f} .
$$

We denote

$$
\widetilde{\boldsymbol{\omega}}=P_{f}^{\mathrm{div}}\left(\boldsymbol{\omega}_{*} \circ \Phi_{f}^{-1}\right), \quad \widetilde{\boldsymbol{\xi}}=P_{f}^{\mathrm{div}}\left(\boldsymbol{\xi}_{*} \circ \Phi_{f}^{-1}\right) .
$$

It follows from Lemma 2.1 for harmonic coordinates and standard elliptic estimates that

$$
\|(\widetilde{\omega}, \widetilde{\xi})\|_{H^{s-1}\left(\Omega_{f}\right)} \leq C\left(M_{1}\right), \quad\left\|\left(\partial_{t} \widetilde{\omega}, \partial_{t} \widetilde{\xi}\right)\right\|_{H^{s-2}\left(\Omega_{f}\right)} \leq C\left(M_{1}, M_{2}\right) .
$$

Moreover, since $\operatorname{div} \widetilde{\omega}=0$ and $\widetilde{\omega}_{3}=\mathbf{e}_{3} \cdot \widetilde{\omega}=\mathbf{e}_{3} \cdot \omega_{*}=\omega_{* 3}$ on $\Gamma$, $\widetilde{\omega}$ satisfies compatibility conditions in (4.2) according to 6.7). Similar argument applies to $\widetilde{\xi}$. Then we can define the velocity field $\mathbf{u}$ and magnetic field $\mathbf{h}$ in $\Omega_{f}$ by solving the following div-curl system

$$
\left\{\begin{array}{l}
\operatorname{curl} \mathbf{u}=\widetilde{\omega}, \operatorname{div} \mathbf{u}=0 \text { in } \Omega_{f} \\
\mathbf{u} \cdot \mathbf{N}_{f}=\partial_{t} f \text { on } \Gamma_{f}, \mathbf{u} \cdot \mathbf{e}_{3}=0, \int_{\Gamma} u_{i} d x^{\prime}=\beta_{i} \text { on } \Gamma, i=1,2
\end{array}\right.
$$

and

$$
\left\{\begin{array}{l}
\operatorname{curl} \mathbf{h}=\widetilde{\boldsymbol{\xi}}, \operatorname{div} \mathbf{h}=0 \text { in } \Omega_{f}, \\
\mathbf{h} \cdot \mathbf{N}_{f}=0 \text { on } \Gamma_{f}, \mathbf{h} \cdot \mathbf{e}_{3}=0, \int_{\Gamma} h_{i} d x^{\prime}=\gamma_{i} \text { on } \Gamma, i=1,2 .
\end{array}\right.
$$

It follows from Proposition 4.1 and 6.11) that

$$
\begin{aligned}
&\|\mathbf{u}\|_{H^{s}\left(\Omega_{f}\right)} \leq C\left(M_{1}\right)\left(\|\widetilde{\omega}\|_{H^{s-1}\left(\Omega_{f}\right)}+\left\|\partial_{t} f\right\|_{H^{s-\frac{1}{2}}}+\left|\beta_{1}\right|+\left|\beta_{2}\right|\right) \leq C\left(M_{1}\right), \\
&\|\mathbf{h}\|_{H^{s}\left(\Omega_{f}\right)} \leq C\left(M_{1}\right)\left(\|\widetilde{\boldsymbol{\xi}}\|_{H^{s-1}\left(\Omega_{f}\right)}+\left|\gamma_{1}\right|+\left|\gamma_{2}\right|\right) \leq C\left(M_{1}\right) .
\end{aligned}
$$

In addition,

$$
\mathbf{u}(0)=\mathbf{u}_{0}, \mathbf{h}(0)=\mathbf{h}_{0}
$$


Using the fact that on $\Gamma_{f}$,

$$
\partial_{t}\left(\mathbf{u} \cdot \mathbf{N}_{f}\right)=\left(\partial_{t} \mathbf{u}+\partial_{3} \mathbf{u} \partial_{t} f\right) \cdot \mathbf{N}_{f}+\mathbf{u} \cdot \partial_{t} \mathbf{N}_{f},
$$

we deduce that

$$
\left\{\begin{array}{l}
\operatorname{curl} \partial_{t} \mathbf{u}=\partial_{t} \widetilde{\boldsymbol{\omega}}, \operatorname{div} \partial_{t} \mathbf{u}=0 \text { in } \Omega_{f}, \\
\partial_{t} \mathbf{u} \cdot \mathbf{N}_{f}=\partial_{t}^{2} f-\partial_{t} f \partial_{3} \mathbf{u} \cdot \mathbf{N}_{f}+u_{1} \partial_{1} \partial_{t} f+u_{2} \partial_{2} \partial_{t} f \text { on } \Gamma_{f}, \\
\partial_{t} \mathbf{u} \cdot \mathbf{e}_{3}=0, \int_{\Gamma} \partial_{t} u_{i} d x=\partial_{t} \beta_{i} \text { on } \Gamma, i=1,2 .
\end{array}\right.
$$

By Proposition 4.1 and 6.11 again,

$$
\left\|\partial_{t} \mathbf{u}\right\|_{H^{s-1}\left(\Omega_{f}\right)} \leq C\left(M_{1}, M_{2}\right),
$$

which implies

$$
\|\mathbf{u}(t)\|_{L^{\infty}\left(\Gamma_{f}\right)} \leq\left\|\mathbf{u}_{0}\right\|_{L^{\infty}\left(\Gamma_{f_{0}}\right)}+\int_{0}^{t}\left\|\partial_{t} \mathbf{u}\right\|_{L^{\infty}\left(\Gamma_{f}\right)} d t \leq M_{0}+T C\left(M_{1}, M_{2}\right) .
$$

Similarly,

$$
\left\|\partial_{t} \mathbf{h}(t)\right\|_{H^{s-1}\left(\Omega_{f}\right)} \leq C\left(M_{1}, M_{2}\right), \quad\|\mathbf{h}(t)\|_{L^{\infty}\left(\Gamma_{f}\right)} \leq M_{0}+T C\left(M_{1}, M_{2}\right) .
$$

Also, we have

$$
\left\|f(t)-f_{0}\right\|_{L^{\infty}} \leq\left\|f(t)-f_{0}\right\|_{H^{s-\frac{1}{2}}} \leq T\left\|\partial_{t} f\right\|_{H^{s-\frac{1}{2}}} \leq T M_{1}
$$

as well as

$$
\left|\Lambda(\mathbf{h}, \hat{\mathbf{h}})-\Lambda\left(\mathbf{h}_{0}, \hat{\mathbf{h}}_{0}\right)\right| \leq T C\left(\left\|\partial_{t} \mathbf{h}\right\|_{L^{\infty}\left(\Gamma_{f}\right)},\left\|\partial_{t} \hat{\mathbf{h}}\right\|_{L^{\infty}\left(\Gamma_{f}\right)}\right) \leq T C\left(M_{0}, M_{1}, M_{2}\right) .
$$

Without loss of generality, we assume $M_{1} \leq C\left(M_{1}\right) \leq C\left(M_{0}, M_{1}\right) \leq C\left(M_{1}, M_{2}\right) \leq$ $C\left(M_{0}, M_{1}, M_{2}\right)$ and choose $T \leq \min \left\{1, T_{0}\right\}$ small enough so that

$$
T C\left(M_{0}, M_{1}, M_{2}\right) \leq \min \left\{\delta_{0}, c_{1}\right\}\left(\leq M_{0}\right) .
$$

Let $L_{0}=2 M_{0}, L_{1}=10 C\left(M_{0}, M_{1}\right), L_{2}=10 C\left(M_{0}, M_{1}, M_{2}\right)$. We conclude that for any $t \in[0, T]$,

- $\|(\mathbf{u}, \mathbf{h}, \hat{\mathbf{h}})(t)\|_{L^{\infty}\left(\Gamma_{f}\right)} \leq L_{0}$

- $\|f(t)\|_{H^{s+\frac{1}{2}}}+\left\|\partial_{t} f(t)\right\|_{H^{s-\frac{1}{2}}}+\|(\mathbf{u}, \mathbf{h})(t)\|_{H^{s}\left(\Omega_{f}\right)}+\|\hat{\mathbf{h}}(t)\|_{H^{s}\left(\Omega_{f}^{+}\right)}+\left\|\partial_{t} \hat{\mathbf{h}}(t)\right\|_{H^{s-1}\left(\Omega_{f}^{+}\right)} \leq L_{1}$;

- $\left\|\left(\partial_{t} \mathbf{u}, \partial_{t} \mathbf{h}\right)(t)\right\|_{L^{\infty}\left(\Gamma_{f}\right)} \leq L_{2}$

- $-\left(1-c_{0}\right) \leq f\left(t, x^{\prime}\right) \leq\left(1-c_{0}\right),\left\|f(t)-f_{*}\right\|_{H^{s-\frac{1}{2}}} \leq \delta_{0}$;

- $\Lambda(\mathbf{h}, \hat{\mathbf{h}})(t) \geq c_{1}$;

which are nothing but the bounds listed at the beginning of Section 5 .

6.2. Define the iteration map. Given $(f, \mathbf{u}, \mathbf{h}, \hat{\mathbf{h}})$ as above, we define the iteration map. We first solve $\bar{f}_{1}$ by the linearized system (5.3) and then $(\overline{\boldsymbol{\omega}}, \overline{\boldsymbol{\xi}})$ by $(5.8)$ and (5.9) with the initial data

$$
\left(\bar{f}_{1}(0), \bar{\theta}(0), \overline{\boldsymbol{\omega}}(0), \overline{\boldsymbol{\xi}}(0)\right)=\left(f_{0},\left(\partial_{t} f\right)_{I}, \boldsymbol{\omega}_{* I}, \boldsymbol{\xi}_{* I}\right)
$$


We define

$$
\begin{aligned}
& \overline{\boldsymbol{\omega}}_{*}=\overline{\boldsymbol{\omega}} \circ \Phi_{f}, \overline{\boldsymbol{\xi}}_{*}=\overline{\boldsymbol{\xi}} \circ \Phi_{f}, \\
& \bar{\beta}_{i}(t)=\beta_{i}(0)-\int_{0}^{t} \int_{\Gamma}\left(u_{j} \partial_{j} u_{i}-h_{j} \partial_{j} h_{i}\right) d x^{\prime} d \tau, \\
& \bar{\gamma}_{i}(t)=\gamma_{i}(0)-\int_{0}^{t} \int_{\Gamma}\left(u_{j} \partial_{j} h_{i}-h_{j} \partial_{j} u_{i}\right) d x^{\prime} d \tau, \\
& \bar{f}\left(t, x^{\prime}\right)=\bar{f}_{1}\left(t, x^{\prime}\right)-\left\langle\bar{f}_{1}\right\rangle+\left\langle f_{0}\right\rangle .
\end{aligned}
$$

Note that $\langle\bar{f}\rangle=\left\langle f_{0}\right\rangle$ and $\int_{\mathbb{T}^{2}} \partial_{t} \bar{f}\left(t, x^{\prime}\right) d x^{\prime}=0$ for $t \in[0, T]$. Moreover, according to Proposition 5.2,

$$
\int_{\Gamma} \overline{\boldsymbol{\omega}}_{* 3} d x^{\prime}=\int_{\Gamma} \overline{\boldsymbol{\omega}}_{3} d x^{\prime}=0, \quad \int_{\Gamma} \overline{\boldsymbol{\xi}}_{* 3} d x^{\prime}=\int_{\Gamma} \overline{\boldsymbol{\xi}}_{3} d x^{\prime}=0 .
$$

The iteration map $\mathcal{F}$ is defined as follows

$$
\mathcal{F}\left(f, \omega_{*}, \boldsymbol{\xi}_{*}, \beta_{i}, \gamma_{i}\right) \stackrel{\text { def }}{=}\left(\bar{f}, \overline{\boldsymbol{\omega}}_{*}, \overline{\boldsymbol{\xi}}_{*}, \bar{\beta}_{i}, \bar{\gamma}_{i}\right) .
$$

Proposition 6.1. There exist $M_{1}, M_{2}, T>0$ depending on $c_{0}, c_{1}, \delta_{0}, M_{0}$ so that $\mathcal{F}$ is a map from $X\left(T, M_{1}, M_{2}\right)$ to itself.

Proof. First note that the initial conditions is automatically satisfied. Proposition 5.1 and Proposition 5.2 ensure that for any $t \in[0, T]$,

$$
\left(\|\bar{f}(t)\|_{H^{s+\frac{1}{2}}}+\left\|\partial_{t} \bar{f}(t)\right\|_{H^{s-\frac{1}{2}}}+\left\|\overline{\boldsymbol{\omega}}_{*}(t)\right\|_{H^{s-1}\left(\Omega_{*}\right)}+\left\|\overline{\boldsymbol{\xi}}_{*}(t)\right\|_{H^{s-1}\left(\Omega_{*}\right)}\right) \leq C\left(c_{0}, M_{0}\right) e^{C\left(M_{1}, M_{2}\right) T} .
$$

From the equation (5.3), (5.8) and (5.9) together with (6.9) for $\hat{\mathbf{h}}$, we deduce that

$$
\sup _{t \in[0, T]}\left(\left\|\partial_{t}^{2} \bar{f}\right\|_{H^{s-\frac{3}{2}}}+\left\|\left(\partial_{t} \omega_{*}, \partial_{t} \xi_{*}\right)\right\|_{H^{s-2}\left(\Omega_{*}\right)}\right) \leq C\left(M_{1}\right) .
$$

Moreover, it is obvious that

$$
\begin{aligned}
& \left|\partial_{t} \bar{\beta}_{i}(t)\right|+\left|\partial_{t} \bar{\gamma}_{i}(t)\right| \leq C\left(M_{1}\right), \\
& \left|\bar{\beta}_{i}(t)\right|+\left|\bar{\gamma}_{i}(t)\right| \leq M_{0}+T C\left(M_{1}\right), \\
& \left\|\bar{f}(t)-f_{*}\right\|_{H^{s-\frac{1}{2}}} \leq \int_{0}^{t}\left\|\partial_{t} \bar{f}(\tau)\right\|_{H^{s-\frac{1}{2}}} d \tau \leq T C\left(M_{1}\right) .
\end{aligned}
$$

We take $M_{1}=2 \max \left\{M_{0}, C\left(c_{0}, M_{0}\right)\right\}$ and $M_{2}=C\left(M_{1}\right)$. Finally, let $T$ be sufficiently small depending only on $c_{0}, c_{1}, \delta_{0}, M_{0}$ so that all other conditions in Definition 6.1 are satisfied.

\section{Contraction of the Iteration Map}

7.1. Contraction. Let $\left(f^{A}, \omega_{*}^{A}, \boldsymbol{\xi}_{*}^{A}, \beta_{i}^{A}, \gamma_{i}^{A}\right)$ and $\left(f^{B}, \omega_{*}^{B}, \boldsymbol{\xi}_{*}^{B}, \beta_{i}^{B}, \gamma_{i}^{B}\right)$ be two elements in $\mathcal{X}\left(T, M_{1}, M_{2}\right)$, and $\left(\bar{f}^{C}, \bar{\omega}_{*}^{C}, \overline{\boldsymbol{\xi}}_{*}^{C}, \bar{\beta}_{i}^{C}, \bar{\gamma}_{i}^{C}\right)=\mathcal{F}\left(f^{C}, \omega_{*}^{C}, \xi_{*}^{C}, \beta_{i}^{C}, \gamma_{i}^{C}\right)$ for $C=A, B$. Correspondingly, we have quantities $\mathbf{u}^{C}, \mathbf{h}^{C}$ and $\hat{\mathbf{h}}^{C}$ defined in $\Omega_{f^{C}}$ and $\Omega_{f C}^{+}$respectively. For a quantity $q$, we denote by $q^{D}$ the difference $q^{A}-q^{B}$. 
Proposition 7.1. There exists $T>0$ depending on $c_{0}, \delta_{0}, M_{0}$ so that

$$
\begin{aligned}
\bar{E}_{s}^{D}:=\sup _{t \in[0, T]}( & \left\|\bar{f}^{D}(t)\right\|_{H^{s-\frac{1}{2}}}+\left\|\partial_{t} \bar{f}^{D}(t)\right\|_{H^{s-\frac{3}{2}}}+\left\|\overline{\boldsymbol{\omega}}_{*}^{D}(t)\right\|_{H^{s-2}\left(\Omega_{*}\right)} \\
& \left.+\left\|\overline{\boldsymbol{\xi}}_{*}^{D}(t)\right\|_{H^{s-2}\left(\Omega_{*}\right)}+\left|\bar{\beta}_{i}^{D}(t)\right|+\left|\bar{\gamma}_{i}^{D}(t)\right|\right) \\
\leq \frac{1}{2} \sup _{t \in[0, T]} & \left(\left\|f^{D}(t)\right\|_{H^{s-\frac{1}{2}}}+\left\|\partial_{t} f^{D}(t)\right\|_{H^{s-\frac{3}{2}}}+\left\|\omega_{*}^{D}(t)\right\|_{H^{s-2}\left(\Omega_{*}\right)}\right. \\
& \left.+\left\|\boldsymbol{\xi}_{*}^{D}(t)\right\|_{H^{s-2}\left(\Omega_{*}\right)}+\left|\beta_{i}^{D}(t)\right|+\left|\gamma_{i}^{D}(t)\right|\right):=E_{s}^{D} .
\end{aligned}
$$

Proof. First of all, by the elliptic estimates, we have

$$
\left\|\Phi_{f^{A}}-\Phi_{f^{B}}\right\|_{H^{s}\left(\Omega_{*}\right)} \leq C\left(M_{1}\right)\left\|f^{A}-f^{B}\right\|_{H^{s-\frac{1}{2}}} \leq C E_{s}^{D} .
$$

Due to the fact that $\mathbf{u}^{A}$ and $\mathbf{u}^{B}$ are defined on different regions, one can not estimate their difference directly. To this end, we introduce for $C=A, B$,

$$
\mathbf{u}_{*}^{C}=\mathbf{u}^{C} \circ \Phi_{f^{C}}, \mathbf{h}_{*}^{C}=\mathbf{h}^{C} \circ \Phi_{f}, \hat{\mathbf{h}}_{*}^{C}=\hat{\mathbf{h}}^{C} \circ \Phi_{f^{C}}^{+} .
$$

We first show that

$$
\left\|\left(\mathbf{u}_{*}^{D}, \mathbf{h}_{*}^{D}\right)\right\|_{H^{s-1}\left(\Omega_{*}\right)}+\left\|\hat{\mathbf{h}}_{*}^{D}\right\|_{H^{s-1}\left(\Omega_{*}^{+}\right)} \leq C E_{s}^{D} .
$$

For a vector field $\mathbf{v}_{*}$ defined on $\Omega_{*}$, we define

$$
\operatorname{curl}_{C} \mathbf{v}_{*}=\left(\operatorname{curl}\left(\mathbf{v}_{*} \circ\left(\Phi_{f^{C}}\right)^{-1}\right)\right) \circ \Phi_{f}, \operatorname{div}_{C} \mathbf{v}_{*}=\left(\operatorname{div}\left(\mathbf{v}_{*} \circ\left(\Phi_{f^{C}}\right)^{-1}\right) \circ \Phi_{f^{C}},\right.
$$

for $C=A, B$. Then we find by (6.12) that for $C=A, B$,

$$
\left\{\begin{array}{l}
\operatorname{curl}_{C} \mathbf{u}_{*}^{C}=\widetilde{\omega}_{*}^{C}, \operatorname{div}_{C} \mathbf{u}_{*}^{C}=0 \text { in } \Omega_{*}, \\
\mathbf{u}_{*}^{C} \cdot \mathbf{N}_{f}=\partial_{t} f^{C} \text { on } \Gamma_{*}, \mathbf{u}^{C} \cdot \mathbf{e}_{3}=0, \int_{\Gamma} u_{i}^{C} d x^{\prime}=\beta_{i}^{C} \text { on } \Gamma .
\end{array}\right.
$$

Thus, we obtain

$$
\left\{\begin{array}{l}
\operatorname{curl}_{A} \mathbf{u}_{*}^{D}=\widetilde{\omega}_{*}^{D}+\left(\operatorname{curl}_{B}-\operatorname{curl}_{A}\right) \mathbf{u}_{*}^{B} \text { in } \Omega_{*}, \\
\operatorname{div}_{A} \mathbf{u}_{*}^{D}=\left(\operatorname{div}_{B}-\operatorname{div}_{A}\right) \mathbf{u}_{*}^{B} \text { in } \Omega_{*}, \\
\mathbf{u}_{*}^{D} \cdot \mathbf{N}_{f^{A}}=\partial_{t} f^{D}+\mathbf{u}_{*}^{B} \cdot\left(\mathbf{N}_{f^{B}}-\mathbf{N}_{f^{A}}\right) \text { on } \Gamma_{*}, \\
\mathbf{u}_{*}^{D} \cdot \mathbf{e}_{3}=0, \int_{\Gamma} u_{i}^{D} d x^{\prime}=\beta_{i}^{D} \text { on } \Gamma .
\end{array}\right.
$$

A tedious but direct calculation shows that

$$
\left\|\left(\operatorname{curl}_{B}-\operatorname{curl}_{A}\right) \mathbf{u}_{*}^{B}\right\|_{H^{s-2}\left(\Omega_{*}\right)} \leq C\left\|\Phi_{f^{A}}-\Phi_{f^{B}}\right\|_{H^{s-1}\left(\Omega_{*}\right)} \leq C\left\|f^{D}\right\|_{H^{s-\frac{1}{2}}} \leq C E_{s}^{D} .
$$

Similarly,

$$
\left\|\left(\operatorname{div}_{B}-\operatorname{div}_{A}\right) \mathbf{u}_{*}^{B}\right\|_{H^{s-2}\left(\Omega_{*}\right)} \leq C E_{s}^{D}, \quad\left\|\mathbf{u}_{*}^{B} \cdot\left(\mathbf{N}_{f^{B}}-\mathbf{N}_{f^{B}}\right)\right\|_{H^{s-\frac{3}{2}}} \leq C E_{s}^{D} .
$$

We deduce from Proposition 4.1 that

$$
\left\|\mathbf{u}_{*}^{D}\right\|_{H^{s-1}\left(\Omega_{*}\right)} \leq C\left(\left\|\widetilde{\omega}_{*}^{D}\right\|_{H^{s-2}\left(\Omega_{*}\right)}+\left\|\partial_{t} f^{D}\right\|_{H^{s-\frac{3}{2}}}+E^{D}\right) \leq C E_{s}^{D} .
$$

By applying similar arguments to $\mathbf{h}, \hat{\mathbf{h}}$, we have from Proposition 4.1 4.2 that

$$
\left\|\mathbf{h}_{*}^{D}\right\|_{H^{s-1}\left(\Omega_{*}\right)} \leq C E_{s}^{D}, \quad\left\|\hat{\mathbf{h}}_{*}^{D}\right\|_{H^{s-1}\left(\Omega_{*}^{+}\right)} \leq C E_{s}^{D} .
$$


Thus, we conclude the proof of (7.1).

To estimate $f^{D}$, we first note that

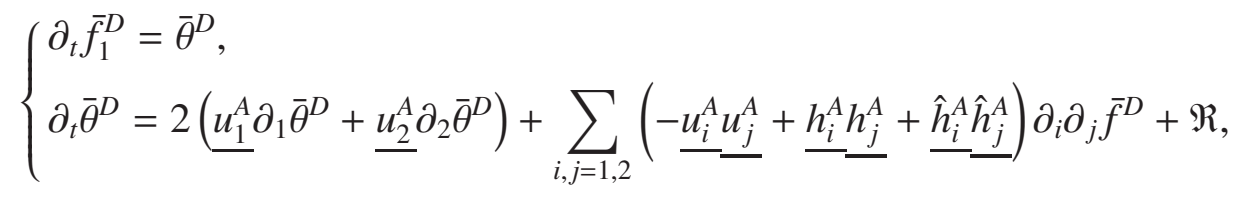

where

$$
\begin{aligned}
\Re= & -2\left(\left(\underline{u_{1}^{D}}+\underline{u_{1}^{D}}\right) \partial_{1} \bar{\theta}^{B}+\left(\underline{u_{2}^{D}}+\underline{u_{2}^{D}}\right) \partial_{2} \bar{\theta}^{B}\right) \\
& +\sum_{i, j=1,2}\left(\left(-\underline{u_{i}^{A}} \underline{u_{j}^{A}}+\underline{h_{i}^{A}} \underline{h_{j}^{A}}+\underline{\hat{h}_{i}^{A}} \underline{\hat{h}_{j}^{A}}\right)-\left(-\underline{u_{i}^{B}} \underline{u_{j}^{B}}+\underline{h_{i}^{B}} \underline{h_{j}^{B}}+\underline{\hat{h}_{i}^{B}} \underline{\hat{h}_{j}^{B}}\right)\right) \partial_{i} \partial_{j} \bar{f}_{1}^{B} \\
& +\mathfrak{g}^{A}-\mathfrak{g}^{B} .
\end{aligned}
$$

Here for $C=A, B$,

$\mathfrak{g}^{C}=-\frac{1}{2} \mathbf{N}_{f^{C}} \cdot \underline{\nabla\left(p_{\mathbf{u}^{C}, \mathbf{u}^{C}}-p_{\mathbf{h}^{C}, \mathbf{h}^{C}}\right)}+\frac{1}{2} \mathbf{N}_{f^{C}} \cdot \underline{\nabla\left(\left|\hat{\mathbf{h}}^{C}\right|^{2}-\hat{\mathcal{H}}_{f^{C}}^{+}\left|\hat{\mathbf{h}}^{C}\right|^{2}\right)}+\frac{1}{2}\left(\hat{\mathcal{N}}_{f^{C}}^{+}-\mathcal{N}_{f}\right)\left|\hat{\mathbf{h}}^{C}\right|^{2}$.

It is direct to verify that

$$
\|\Re\|_{H^{s-\frac{3}{2}}} \leq C E_{s}^{D} .
$$

We denote

$$
\begin{aligned}
\bar{F}_{s}^{D}\left(\partial_{t} \bar{f}_{1}^{D}, \bar{f}_{1}^{D}\right)= & \left\|\left(\partial_{t}+u_{i}^{A} \partial_{i}\right)\langle\nabla\rangle^{s-\frac{3}{2}} \bar{f}_{1}^{D}\right\|_{L^{2}}^{2}+\frac{1}{2}\left\|h_{i}^{A} \partial_{i}\langle\nabla\rangle^{s-\frac{3}{2}} \bar{f}_{1}^{D}\right\|_{L^{2}}^{2} \\
& +\frac{1}{2}\left\|\hat{h}_{i}^{A} \partial_{i}\langle\nabla\rangle^{s-\frac{3}{2}} \bar{f}_{1}^{D}\right\|_{L^{2}}^{2} .
\end{aligned}
$$

A similar argument as in Proposition 5.1 gives

$$
\frac{d}{d t}\left(\bar{F}_{s}^{D}\left(\partial_{t} \bar{f}_{1}^{D}, \bar{f}_{1}^{D}\right)+\left\|\bar{f}_{1}^{D}\right\|_{L^{2}}^{2}+\left\|\partial_{t} \bar{f}_{1}^{D}\right\|_{L^{2}}^{2}\right) \leq C\left(E_{s}^{D}+\bar{E}_{1 s}^{D}\right)
$$

where

$$
\bar{E}_{1 s}^{D}=\sup _{t \in[0, T]}\left(\left\|\bar{f}_{1}^{D}(t)\right\|_{H^{s-\frac{1}{2}}}+\left\|\partial_{t} \bar{f}_{1}^{D}(t)\right\|_{H^{s-\frac{3}{2}}}\right) .
$$

Stability condition on $\mathbf{h}^{A}, \hat{\mathbf{h}}^{A}$ implies that

$$
\left\|\bar{f}_{1}^{D}\right\|_{H^{s-\frac{1}{2}}}^{2}+\left\|\partial_{t} \bar{f}_{1}^{D}\right\|_{H^{s-\frac{3}{2}}}^{2} \leq C\left(\bar{F}_{s}^{D}\left(\bar{f}_{1}^{D}, \partial_{t} \bar{f}_{1}^{D}\right)+\left\|\bar{f}_{1}^{D}\right\|_{L^{2}}^{2}+\left\|\partial_{t} \bar{f}_{1}^{D}\right\|_{L^{2}}^{2}\right) .
$$

It follows that

$$
\bar{E}_{1 s} \leq C T E_{s}^{D}
$$

which implies

$$
\sup _{t \in[0, T]}\left(\left\|\bar{f}^{D}(t)\right\|_{H^{s-1}}+\left\|\partial_{t} \bar{f}^{D}(t)\right\|_{H^{s-\frac{3}{2}}}\right) \leq C T E_{s}^{D} .
$$

Similar to the proof of Proposition 5.2, one can show that

$$
\sup _{t \in[0, T]}\left(\left\|\bar{\omega}_{*}^{D}(t)\right\|_{H^{s-2}\left(\Omega_{*}\right)}+\left\|\bar{\xi}_{*}^{D}\right\|_{H^{s-2}\left(\Omega_{*}\right)}\right) \leq C T E_{s}^{D} .
$$


Finally, by using the equation

$$
\bar{\beta}_{i}^{C}(t)=\beta_{i}^{C}(0)+\int_{0}^{t} \int_{\Gamma} u_{j}^{C}\left(\partial_{j} u_{i}^{C}-h_{j}^{C} \partial_{j} h_{i}^{C}\right) d x^{\prime} d \tau,
$$

it is obvious that

$$
\left|\bar{\beta}_{i}^{D}(t)\right| \leq\left|\beta_{i I}^{D}\right|+C T E_{s}^{D}=C T E_{s}^{D} .
$$

Similarly,

$$
\left|\bar{\gamma}_{i}^{D}(t)\right| \leq\left|\gamma_{i I}^{D}\right|+C T E_{s}^{D}=C T E_{s}^{D} .
$$

We deduce from (7.1) and (7.3)-(7.6) that

$$
\bar{E}_{s}^{D} \leq C T E_{s}^{D} .
$$

The proof is concluded by taking $T=\frac{1}{2 C}$ with $C$ depending only on $c_{0}, \delta_{0}, M_{0}$.

7.2. The limit system. Proposition 6.1 and Proposition 7.1 ensure that the map $\mathcal{F}$ has a unique fixed point $\left(f, \omega, \xi, \beta_{i}, \gamma_{i}\right)$ in $\mathcal{X}\left(T, M_{1}, M_{2}\right)$. From the construction of $\mathcal{F}$, we know that $\left(f, \omega, \xi, \beta_{i}, \gamma_{i}\right)$ satisfies

$$
\left\{\begin{aligned}
\partial_{t} f= & \theta-\langle\theta\rangle, \\
\partial_{t} \theta= & 2\left(\underline{u_{1}} \partial_{1} \theta+\underline{u_{2}} \partial_{2} \theta\right)-\sum_{i, j=1,2}\left(\underline{u_{i}} \underline{u_{j}}-\underline{h_{i}} \underline{h_{j}}-\underline{\hat{h}_{i}} \underline{\hat{h}_{j}}\right) \partial_{i} \partial_{j} f \\
& -\mathbf{N}_{f} \cdot \underline{\nabla\left(p_{\mathbf{u}, \mathbf{u}}-p_{\mathbf{h}, \mathbf{h}}\right)}+\frac{1}{2} \mathbf{N}_{f} \cdot \underline{\nabla\left(|\hat{\mathbf{h}}|^{2}-\hat{\mathcal{H}}_{f}^{+}|\hat{\mathbf{h}}|^{2}\right)}+\frac{1}{2}\left(\hat{\mathcal{N}}_{f}^{+}-\mathcal{N}_{f}\right)|\hat{\mathbf{h}}|^{2},
\end{aligned}\right.
$$

where $(\mathbf{u}, \mathbf{h})$ solves the div-curl system

$$
\left\{\begin{array}{l}
\operatorname{curl} \mathbf{u}=P_{f}^{d i v} \omega, \operatorname{div} \mathbf{u}=0 \text { in } \Omega_{f} \\
\mathbf{u} \cdot \mathbf{N}_{f}=\partial_{t} f \text { on } \Gamma_{f}, u_{3}=0 \text { on } \Gamma \\
\int_{\Gamma} u_{i} d x^{\prime}=\beta_{i}, \partial_{t} \beta_{i}=-\int_{\Gamma}\left(u_{j} \partial_{j} u_{i}-h_{j} \partial_{j} h_{i}\right) d x^{\prime}, i=1,2,
\end{array}\right.
$$

and

$$
\left\{\begin{array}{l}
\operatorname{curl} \mathbf{h}=P_{f}^{d i v} \boldsymbol{\xi}, \operatorname{div} \mathbf{h}=0 \text { in } \Omega_{f}, \\
\mathbf{h} \cdot \mathbf{N}_{f}=0 \text { on } \Gamma_{f}, h_{3}=0 \text { on } \Gamma, \\
\int_{\Gamma} h_{i} d x^{\prime}=\gamma_{i}, \partial_{t} \gamma_{i}=-\int_{\Gamma}\left(u_{j} \partial_{j} h_{i}-h_{j} \partial_{j} u_{i}\right) d x^{\prime}, i=1,2,
\end{array}\right.
$$

and $\hat{\mathbf{h}}$ solves the div-curl system

$$
\left\{\begin{array}{l}
\operatorname{curl} \hat{\mathbf{h}}=0, \operatorname{div} \hat{\mathbf{h}}=0 \text { in } \Omega_{f}^{+}, \\
\hat{\mathbf{h}} \cdot \mathbf{N}_{f}=0 \text { on } \Gamma_{f}, \hat{\mathbf{h}} \times \mathbf{e}_{3}=\hat{\mathbf{J}} \text { on } \Gamma^{+},
\end{array}\right.
$$

as well as

$$
\left\{\begin{array}{l}
\partial_{t} \boldsymbol{\omega}+\mathbf{u} \cdot \nabla \boldsymbol{\omega}=\mathbf{h} \cdot \nabla \boldsymbol{\xi}+\boldsymbol{\omega} \cdot \nabla \mathbf{u}-\boldsymbol{\xi} \cdot \nabla \mathbf{h} \text { in } Q_{T}, \\
\partial_{t} \boldsymbol{\xi}+\mathbf{u} \cdot \nabla \boldsymbol{\xi}=\mathbf{h} \cdot \nabla \boldsymbol{\omega}+\boldsymbol{\xi} \cdot \nabla \mathbf{u}-\boldsymbol{\omega} \cdot \nabla \mathbf{h}-2 \nabla u_{i} \times \nabla h_{i} \text { in } Q_{T} .
\end{array}\right.
$$

We also recall that $p_{\mathbf{v}, \mathbf{v}}$ for $\mathbf{v}=\mathbf{u}, \mathbf{h}$ is determined by the elliptic equation

$$
\left\{\begin{array}{l}
\Delta p_{\mathbf{v}, \mathbf{v}}=-\operatorname{tr}(\nabla \mathbf{v} \nabla \mathbf{v}) \text { in } \Omega_{f}, \\
p_{\mathbf{v}, \mathbf{v}}=0 \text { on } \Gamma_{f}, \mathbf{e}_{3} \cdot \nabla p_{\mathbf{v}, \mathbf{v}}=0 \text { on } \Gamma .
\end{array}\right.
$$




\section{From the Limit system to the Plasma-VacuUm interface System}

It is not obvious whether the limit system (7.7)-(7.12) is equivalent to the plasmavacuum interface system (1.1)-(1.10). Following [17], we split the proof into several steps.

Step 1. curl $\mathbf{u}=\omega$ and $\operatorname{curl} \mathbf{h}=\boldsymbol{\xi}$.

By $\operatorname{div} \mathbf{u}=\operatorname{div} \mathbf{h}=0$, it is easy to verify that

$$
\begin{aligned}
& \partial_{t} \operatorname{div} \boldsymbol{\omega}+\mathbf{u} \cdot \nabla \operatorname{div} \boldsymbol{\omega}=\mathbf{h} \cdot \nabla \operatorname{div} \boldsymbol{\xi} \text { in } Q_{T}, \\
& \partial_{t} \operatorname{div} \boldsymbol{\xi}+\mathbf{u} \cdot \nabla \operatorname{div} \boldsymbol{\xi}=\mathbf{h} \cdot \nabla \operatorname{div} \boldsymbol{\omega} \text { in } Q_{T},
\end{aligned}
$$

which imply that $\operatorname{div} \omega=\operatorname{div} \boldsymbol{\xi}=0$ since it is satisfied initially. Hence curl $\mathbf{u}=\omega$, $\operatorname{curl} \mathbf{h}=\boldsymbol{\xi}$ according to (7.8) and (7.9).

Step 2. Determination of the pressure.

Let the pressure $p$ in the plasma region be given by

$$
p=\frac{1}{2} \mathcal{H}|\underline{\hat{\mathbf{h}}}|^{2}+p_{\mathbf{u}, \mathbf{u}}-p_{\mathbf{h}, \mathbf{h}}
$$

From the calculations in Section 3.1 ,

$$
-\mathcal{N}_{f}|\hat{\mathbf{h}}|^{2}=2 \sum_{i, j=1,2} \underline{\hat{h}_{i}} \underline{\hat{h}_{j}} \partial_{i} \partial_{j} f-\mathbf{N}_{f} \cdot \underline{\nabla\left(|\hat{\mathbf{h}}|^{2}-\widehat{\mathcal{H}}_{f}^{+}|\hat{\mathbf{h}}|^{2}\right)}+\left(\widehat{\mathcal{N}}_{f}^{+}-\mathcal{N}_{f}\right)|\hat{\mathbf{h}}|^{2}
$$

see (3.9) and (3.10).

Step 3. The velocity equation.

Let

$$
\mathbf{w}=\partial_{t} \mathbf{u}+\mathbf{u} \cdot \nabla \mathbf{u}-\mathbf{h} \cdot \nabla \mathbf{h}+\nabla p .
$$

We will show that $\mathbf{w}$ satisfies the following homogeneous equations:

$$
\left\{\begin{array}{l}
\operatorname{div} \mathbf{w}=0, \operatorname{curl} \mathbf{w}=0 \text { in } \Omega_{f}, \\
\mathbf{w} \cdot \mathbf{N}_{f}=0 \text { on } \Gamma_{f}, w_{3}=0 \text { on } \Gamma, \int_{\Gamma} w_{i} d x^{\prime}=0, i=1,2,
\end{array}\right.
$$

which implies $\mathbf{w} \equiv 0$, i.e.,

$$
\partial_{t} \mathbf{u}+\mathbf{u} \cdot \nabla \mathbf{u}-\mathbf{h} \cdot \nabla \mathbf{h}+\nabla p=0 \text { in } \Omega_{f} .
$$

First, by the definition of $p$,

$$
\operatorname{div}(-\mathbf{u} \cdot \nabla \mathbf{u}+\mathbf{h} \cdot \nabla \mathbf{h}+\nabla p)=0,
$$

which together with $\operatorname{div} \partial_{t} \mathbf{u}=0$ yields $\operatorname{div} \mathbf{w}=0$ in $\Omega_{f}$. On the other hand, a direct computation by using the equation of $\omega$ shows

$$
\begin{aligned}
\operatorname{curl} \partial_{t} \mathbf{u}=\partial_{t} \operatorname{curl} \mathbf{u}=\partial_{t} \omega & =-\mathbf{u} \cdot \nabla \omega+\mathbf{h} \cdot \nabla \boldsymbol{\xi}+\omega \cdot \nabla \mathbf{u}-\boldsymbol{\xi} \cdot \nabla \mathbf{h} \\
& =\operatorname{curl}(-\mathbf{u} \cdot \nabla \mathbf{u}+\mathbf{h} \cdot \nabla \mathbf{h}+\nabla p) .
\end{aligned}
$$

Thus, we obtain $\operatorname{curl} \mathbf{w}=0$ in $\Omega_{f}$.

Since $u_{3}=0, h_{3}=0$ on $\Gamma$,

$$
w_{3}=\partial_{t} u_{3}+u_{i} \partial_{i} u_{3}-h_{i} \partial_{i} h_{3}-\partial_{3} p=0 \text { on } \Gamma \text {. }
$$


Moreover, according to (7.8),

$$
\int_{\Gamma} w_{i} d x^{\prime}=\int_{\Gamma}\left(\partial_{t} u_{i}+u_{j} \partial_{j} u_{i}-h_{j} \partial_{j} h_{i}\right) d x^{\prime}=0, i=1,2 .
$$

It only leaves the boundary condition of $\mathbf{w}$ on $\Gamma_{f}$ to be proved. To this end, we first define the projection operator $\mathcal{P}: L^{2}\left(\mathbb{T}^{2}\right) \rightarrow L^{2}\left(\mathbb{T}^{2}\right)$ as

$$
\mathcal{P} g=g-\langle g\rangle \text {. }
$$

By conversing the computations in Section 3.1, we find

$$
\begin{aligned}
& \mathcal{P}\left\{-2\left(\underline{u_{1}} \partial_{1} \theta+\underline{u_{2}} \partial_{2} \theta\right)-\mathbf{N}_{f} \cdot \underline{\nabla p}-\sum_{i, j=1,2} \underline{u_{i}} \underline{u_{j}} \partial_{i} \partial_{j} f+\sum_{i, j=1,2} \underline{h_{i}} \underline{h_{j}} \partial_{i} \partial_{j} f\right\} \\
& =-2 \mathcal{P}\left(\underline{u_{1}} \partial_{1} \theta+\underline{u_{2}} \partial_{2} \theta\right)-\frac{1}{2} \mathcal{N}_{f}|\hat{\mathbf{h}}|^{2}-\mathcal{P} \mathbf{N}_{f} \cdot \underline{\nabla\left(p_{\mathbf{u}, \mathbf{u}}-p_{\mathbf{h}, \mathbf{h}}\right)} \\
& -\mathcal{P} \sum_{i, j=1,2} \underline{u_{i}} \underline{u_{j}} \partial_{i} \partial_{j} f+\mathcal{P} \sum_{i, j=1,2} \underline{h_{i}} \underline{h_{j}} \partial_{i} \partial_{j} f \\
& =\mathcal{P}\left\{-2\left(\underline{u_{1}} \partial_{1} \theta+\underline{u_{2}} \partial_{2} \theta\right)-\sum_{i, j=1,2}\left(\underline{u_{i}} \underline{u_{j}}-\underline{h_{i}} \underline{h_{j}}-\underline{\hat{h}_{i}} \underline{\hat{h}_{j}}\right) \partial_{i} \partial_{j} f\right. \\
& \left.-\mathbf{N}_{f} \cdot \underline{\nabla\left(p_{\mathbf{u}, \mathbf{u}}-p_{\mathbf{h}, \mathbf{h}}\right)}-\frac{1}{2} \mathbf{N}_{f} \cdot \underline{\nabla\left(|\hat{\mathbf{h}}|^{2}-\widehat{\mathcal{H}}_{f}^{+}|\hat{\mathbf{h}}|^{2}\right)}+\frac{1}{2}\left(\widehat{\mathcal{N}}_{f}^{+}-\mathcal{N}_{f}\right)|\hat{\mathbf{h}}|^{2}\right\} \\
& =\mathcal{P} \partial_{t} \theta \text {. }
\end{aligned}
$$

Recalling that $\partial_{t} \theta=\partial_{t}^{2} f, \partial_{t} f=\underline{\mathbf{u}} \cdot \mathbf{N}_{f}$, we obtain

$$
\begin{gathered}
\mathcal{P}\left\{\partial_{t}\left(\underline{\mathbf{u}} \cdot \mathbf{N}_{f}\right)+2\left(\underline{u_{1}} \partial_{1}\left(\underline{\mathbf{u}} \cdot \mathbf{N}_{f}\right)+\underline{u_{2}} \partial_{2}\left(\underline{\mathbf{u}} \cdot \mathbf{N}_{f}\right)\right)+\mathbf{N}_{f} \cdot \underline{\nabla p}\right. \\
\left.\left.+\sum_{i, j=1,2} \underline{\left(h_{i}\right.} \underline{h_{j}}-\underline{u_{i}} \underline{u_{j}}\right) \partial_{i} \partial_{j} f\right\}=0,
\end{gathered}
$$

which together with the fact

$$
\partial_{t} \mathbf{N}_{f}=\left(-\partial_{1} \partial_{t} f,-\partial_{2} \partial_{t} f, 0\right)=\left(-\partial_{1}\left(\underline{\mathbf{u}} \cdot \mathbf{N}_{f}\right),-\partial_{2}\left(\underline{\mathbf{u}} \cdot \mathbf{N}_{f}\right), 0\right)
$$

implies

$$
\begin{aligned}
& \mathcal{P}\left\{\left(\underline{\partial_{t} \mathbf{u}}+\underline{\partial_{3} \mathbf{u}} \partial_{t} f\right) \cdot \mathbf{N}_{f}+\left(\underline{u_{1}} \partial_{1}\left(\underline{\mathbf{u}} \cdot \mathbf{N}_{f}\right)+\underline{u_{2}} \partial_{2}\left(\underline{\mathbf{u}} \cdot \mathbf{N}_{f}\right)\right)\right. \\
& \left.+\sum_{i, j=1,2}\left(\underline{h_{i}} \underline{h_{j}}-\underline{u_{i}} \underline{u_{j}}\right) \partial_{i} \partial_{j} f+\mathbf{N}_{f} \cdot \nabla p\right\}=0 .
\end{aligned}
$$

It follows from Lemma 3.1 that

$$
\mathcal{P}\left\{\left.\left(\partial_{t} \mathbf{u}+\mathbf{u} \cdot \nabla \mathbf{u}-\mathbf{h} \cdot \nabla \mathbf{h}+\nabla p\right)\right|_{\Gamma_{f}} \cdot \mathbf{N}_{f}\right\}=0 .
$$

On the other hand, (8.4) and (8.5) imply that

$$
\left.\int_{\mathbb{T}^{2}}\left(\partial_{t} \mathbf{u}+\mathbf{u} \cdot \nabla \mathbf{u}-\mathbf{h} \cdot \nabla \mathbf{h}+\nabla p\right)\right|_{\Gamma_{f}} \cdot \mathbf{N}_{f} d x^{\prime}=0,
$$

which together with $(8.6)$ yields

$$
\mathbf{w} \cdot \mathbf{N}_{f}=\left(\partial_{t} \mathbf{u}+\mathbf{u} \cdot \nabla \mathbf{u}-\mathbf{h} \cdot \nabla \mathbf{h}+\nabla p\right) \cdot \mathbf{N}_{f}=0 \text { on } \Gamma_{f} .
$$

This concludes the proof of 8.3 . 
Step 4. The magnetic field equation.

Let $\mathbf{b}=\partial_{t} \mathbf{h}-\mathbf{h} \cdot \nabla \mathbf{u}+\mathbf{u} \cdot \nabla \mathbf{h}$. It suffices to show that

$$
\left\{\begin{array}{l}
\operatorname{div} \mathbf{b}=0, \operatorname{curl} \mathbf{b}=0 \text { in } \Omega_{f}, \\
\mathbf{b} \cdot \mathbf{N}_{f}=0 \text { on } \Gamma_{f}, b_{3}=0 \text { on } \Gamma, \int_{\Gamma} b_{i} d x^{\prime}=0, i=1,2 .
\end{array}\right.
$$

By using $\mathbf{h} \cdot \mathbf{N}_{f}=0$ on $\Gamma_{f}$, we get

$$
\begin{aligned}
0= & \partial_{t}\left(\underline{\mathbf{h}} \cdot \mathbf{N}_{f}\right)+\underline{u_{1}} \partial_{1}\left(\underline{\mathbf{h}} \cdot \mathbf{N}_{f}\right)+\underline{u_{2}} \partial_{2}\left(\underline{\mathbf{h}} \cdot \mathbf{N}_{f}\right) \\
= & \left(\underline{\partial_{t} \mathbf{h}}+\underline{\partial_{3} \mathbf{h}} \partial_{t} f\right) \cdot \mathbf{N}_{f}+\underline{\mathbf{h}} \cdot \partial_{t} \mathbf{N}_{f}+\left(\underline{u_{1} \partial_{1} \mathbf{h}}+\underline{u_{1} \partial_{3} \mathbf{h}} \partial_{1} f\right) \cdot \mathbf{N}_{f} \\
& +\underline{u_{1}} \underline{\mathbf{h}} \cdot \partial_{1} \mathbf{N}_{f}+\left(\underline{u_{2} \partial_{2} \mathbf{h}}+\underline{u_{2} \partial_{3} \mathbf{h}} \partial_{2} f\right) \cdot \mathbf{N}_{f}+\underline{u_{2}} \underline{\mathbf{h}} \cdot \partial_{2} \mathbf{N}_{f} \\
= & \left(\underline{\partial_{t} \mathbf{h}}+\underline{\mathbf{u} \cdot \nabla \mathbf{h}}\right) \cdot \mathbf{N}_{f}+\partial_{t} f \underline{\partial_{3} \mathbf{h}} \cdot \mathbf{N}_{f}+\underline{\mathbf{h}} \cdot \partial_{t} \mathbf{N}_{f} \\
& +\underline{u_{1}} \underline{\mathbf{h}} \cdot \partial_{1} \mathbf{N}_{f}+\underline{u_{2}} \underline{\mathbf{h}} \cdot \partial_{2} \mathbf{N}_{f}+\left(\underline{u_{1} \partial_{3} \mathbf{h}} \partial_{1} f+\underline{u_{2} \partial_{3} \mathbf{h}} \partial_{2} f-\underline{u_{3} \partial_{3} \mathbf{h}}\right) \cdot \mathbf{N}_{f} \\
= & \left(\underline{\partial_{t} \mathbf{h}}+\underline{\mathbf{u} \cdot \nabla \mathbf{h}}\right) \cdot \mathbf{N}_{f}+\underline{\mathbf{h}} \cdot \partial_{t} \mathbf{N}_{f}+\underline{u_{1}} \underline{\mathbf{h}} \cdot \partial_{1} \mathbf{N}_{f}+\underline{u_{2}} \underline{\mathbf{h}} \cdot \partial_{2} \mathbf{N}_{f} .
\end{aligned}
$$

On the other hand,

$$
\begin{aligned}
& \underline{\mathbf{h}} \cdot \partial_{t} \mathbf{N}_{f}+\underline{u_{1}} \underline{\mathbf{h}} \cdot \partial_{1} \mathbf{N}_{f}+\underline{u_{2}} \underline{\mathbf{h}} \cdot \partial_{2} \mathbf{N}_{f} \\
& =-\underline{h_{1}} \partial_{1}\left(\underline{\mathbf{u}} \cdot \mathbf{N}_{f}\right)-\underline{h_{2}} \partial_{2}\left(\underline{\mathbf{u}} \cdot \mathbf{N}_{f}\right)+\underline{u_{1}} \underline{\mathbf{h}} \cdot \partial_{1} \mathbf{N}_{f}+\underline{u_{2}} \underline{\mathbf{h}} \cdot \partial_{2} \mathbf{N}_{f} \\
& =-\underline{h_{1}}\left(\underline{\partial_{1} \mathbf{u}}+\underline{\partial_{3} \mathbf{u}} \partial_{1} f\right) \cdot \mathbf{N}_{f}-\underline{h_{2}}\left(\underline{\partial_{2} \mathbf{u}}+\underline{\partial_{3} \mathbf{u}} \partial_{2} f\right) \cdot \mathbf{N}_{f} \\
& -\underline{h_{1}} \underline{\mathbf{u}} \cdot \partial_{1} \mathbf{N}_{f}-\underline{h_{2}} \underline{\mathbf{u}} \cdot \partial_{2} \mathbf{N}_{f}-\sum_{i, j=1,2} \underline{u_{i}} \underline{h_{j}} \partial_{i} \partial_{j} f \\
& =\underline{h}_{1}\left(\underline{\partial_{1} \mathbf{u}}+\underline{\partial_{3} \mathbf{u}} \partial_{1} f\right) \cdot \mathbf{N}_{f}-\underline{h_{2}}\left(\underline{\partial_{2} \mathbf{u}}+\underline{\partial_{3} \mathbf{u}} \partial_{2} f\right) \cdot \mathbf{N}_{f} \\
& =-(\underline{\mathbf{h} \cdot \nabla \mathbf{u}}) \cdot \mathbf{N}_{f}-\left(\underline{\partial_{3} \mathbf{u}} \cdot \mathbf{N}_{f}\right)\left(\underline{\mathbf{h}} \cdot \mathbf{N}_{f}\right) \\
& =-(\underline{\mathbf{h} \cdot \nabla \mathbf{u}}) \cdot \mathbf{N}_{f} \text {. }
\end{aligned}
$$

Thus, we deduce that

$$
\left(\partial_{t} \mathbf{h}-\mathbf{h} \cdot \nabla \mathbf{u}+\mathbf{u} \cdot \nabla \mathbf{h}\right) \cdot \mathbf{N}_{f}=0 \text { on } \Gamma_{f}
$$

Moreover,

$$
\operatorname{div}(\mathbf{h} \cdot \nabla \mathbf{u}-\mathbf{u} \cdot \nabla \mathbf{h})=0
$$

together with $\operatorname{div} \partial_{t} \mathbf{h}=0$ implies $\operatorname{div} \mathbf{b}=0$. By (7.11), we have

$$
\begin{aligned}
\operatorname{curl}\left(\partial_{t} \mathbf{h}\right) & =\partial_{t} \boldsymbol{\xi}=\operatorname{curl}\left(-\mathbf{u} \cdot \nabla \boldsymbol{\xi}+\mathbf{h} \cdot \nabla \omega+\boldsymbol{\xi} \cdot \nabla \mathbf{u}-\omega \cdot \nabla \mathbf{h}-2 \nabla u_{i} \times \nabla h_{i}\right) \\
& =\operatorname{curl}(\mathbf{h} \cdot \nabla \mathbf{u}-\mathbf{u} \cdot \nabla \mathbf{h}) .
\end{aligned}
$$

Other boundary conditions in (8.7) follows in a similar manner as in Step 3.

We remark that since $\hat{\mathbf{h}}$ is a secondary variable solved out from $f$ (and $\hat{\mathbf{J}}$ ), it automatically satisfies the equations. Furthermore, stability condition (1.20) has been shown at the end of Section 6.1. Hence, Step 1-Step 4 are enough to ensure that $(\mathbf{u}, \mathbf{h}, \hat{\mathbf{h}}, f, p)$ obtained in Section 7 is a solution of the system (1.1)-1.10). 


\section{AcKNOWLEDGMENT}

Yongzhong Sun is supported by NSF of China under Grant No. 11571167 and the PAPD of Jiangsu Higher Education Institutions. Wei Wang is supported by NSF of China under Grant No. 11501502 and the Fundamental Research Funds for the Central Universities. Zhifei Zhang is partially supported by NSF of China under Grant No. 11425103.

\section{REFERENCES}

[1] T. Alazard, N. Burq and C. Zuily, On the Cauchy problem for gravity water waves, Invent. Math., 198(2014), 71-163.

[2] W. I. Axford, Note on a problem of magnetohydrodynamic stability, Canad. J. Phys., 40(1962), 654-655.

[3] G.-Q. Chen and Y.-G Wang, Existence and stability of compressible current-vortex sheets in three-dimensional magnetohydrodynamics, Arch. Ration. Mech. Anal., 187(2008), 369-408.

[4] C. A. Cheng and S. Shkoller, Solvability and regularity for an elliptic system prescribing the curl, divergence, and partial trace of a vector field on sobolev-class domains, arXiv:1408.2469v1.

[5] J.-F. Coulombel, A. Morando, P. Secchi and P. Trebeschi, A priori estimates for 3D incompressible current-vortex sheets, Comm. Math. Phys., 311(2012), 247-275.

[6] D. Coutand and S. Shkoller, Well-posedness of the free-surface incompressible Euler equations with or without surface tension, J. Amer. Math. Soc., 20(2007), 829-930.

[7] D. G. Ebin, The equations of motion of a perfect fluid with free boundary are not well posed, Comm. Partial Differential Equations, 12(1987), 1175-1201.

[8] J. P. Goedbloed and S. Poedts, Principles of Magnetohydrodynamics with Applications to Laboratory and Astrophysical Plasmas, Cambridge University Press, Cambridge, 2004.

[9] X. Gu and Y. Wang, On the construction of solutions to the free-surface incompressible ideal magnetohydrodynamic equations, arXiv:1609.07013.

[10] C. Hao, On the motion of free interface in ideal incompressible MHD, Arch. Rational Mech. Anal. (2017)

[11] C. Hao and T. Luo, A priori estimates for free boundary problem of incompressible inviscid magnetohydrodynamic flows, Arch. Ration. Mech. Anal., 212(2014), 805-847.

[12] D. Lannes, Well-posedness of the water-waves equations, J. Amer. Math. Soc. 18 (2005), 605654.

[13] H. Lindblad, Well-posedness for the motion of an incompressible liquid with free surface boundary, Ann. Math., 162(2005), 109-194.

[14] A. Morando, Y. Trakhinin and P. Trebeschi, Well-posedness of the linearized plasma-vacuum interface problem in ideal incompressible MHD, Quart. Appl. Math., 72(2014), 549-587.

[15] P. Secchi and Y. Trakhinin, Well-posedness of the plasma-vacuum interface problem, Nonlinearity, 27(2014), 105-169.

[16] J. Shatah and C. Zeng, Geometry and a priori estimates for free boundary problems of the Euler's equation, Comm. Pure Appl. Math., 61(2008), 698-744.

[17] Y. Sun, W. Wang and Z. Zhang, Nonlinear stability of current-vortex sheet to the incompressible MHD equations, to appear in Comm. Pure Appl. Math.

[18] S. I. Syrovatskij, The stability of tangential discontinuities in a magnetohydrodynamic medium, Z. Eksperim. Teoret. Fiz., 24(1953), 622-629.

[19] Y. Trakhinin, Existence of compressible current-vortex sheets: variable coefficients linear analysis, Arch. Ration. Mech. Anal., 177(2005), 331-366.

[20] Y. Trakhinin, The existence of current-vortex sheets in ideal compressible magnetohydrodynamics, Arch. Ration. Mech. Anal., 191(2009), 245-310.

[21] Y. Trakhinin, On the well-posedness of a linearized plasma-vacuum interface problem in ideal compressible MHD, J. Differential Equations, 249(2010), 2577-2599. 
[22] Y.-G. Wang and F. Yu, Stabilization effect of magnetic fields on two-dimensional compressible current-vortex sheets, Arch. Ration. Mech. Anal., 208(2013), 341-389.

[23] S. Wu, Well-posedness in Sobolev spaces of the full water wave problem in 2-D, Invent. Math., 130(1997), 39-72.

[24] S. Wu, Well-posedness in Sobolev spaces of the full water wave problem in 3-D, J. Amer. Math. Soc., 12(1999), 445-495.

[25] P. Zhang and Z. Zhang, On the free boundary problem of three-dimensional incompressible Euler equations, Comm. Pure Appl. Math., 61(2008), 877-940.

Department of Mathematics, Nanjing University, 210093, Nanjing, P. R. China

E-mail address: sunyz@nju.edu.cn

School of Mathematical Sciences, Zhejiang University, 310027, Hangzhou, P. R. China

E-mail address: wangw07@zju.edu.cn

School of Mathematical Sciences, Peking University, 100871, P. R. China

E-mail address: zfzhang@math.pku.edu.cn 